\title{
IR diagnostics of embedded jets: velocity resolved observations of the HH34 and $\mathrm{HH} 1$ jets ${ }^{\star}$
}

\author{
R. Garcia Lopez ${ }^{1,2,4}$, B. Nisini ${ }^{1}$, T. Giannini ${ }^{1}$, J. Eislöffel ${ }^{2}$, F. Bacciotti ${ }^{3}$, and L. Podio $^{3}$ \\ 1 INAF - Osservatorio Astronomico di Roma, via di Frascati 33, 00040 Monteporzio Catone, Italy \\ e-mail: garcia@mporzio.astro.it \\ 2 Thüringer Landessternwarte Tautenburg, Sternwarte 5, 07778 Tautenburg, Germany \\ 3 INAF - Osservatorio Astrofisico di Arcetri, Largo E. Fermi 5, 50125 Florence, Italy \\ 4 Università degli Studi di Roma "Tor Vergata" - Dipartimento di Fisica, via della Ricerca Scientifica 1, 00133 Roma, Italy \\ Received 9 November 2007 / Accepted 18 May 2008
}

ABSTRACT

Context. We present VLT-ISAAC medium resolution spectroscopy of the HH34 and HH1 jets, driven by young Class 0/I sources. Aims. Our aim is to derive the kinematics and the physical parameters associated with infrared jets, and to study how they vary with jet velocity.

Methods. We use several important diagnostic lines covered by our spectral range, such as [Fe II] $1.644 \mu \mathrm{m}, 1.600 \mu \mathrm{m}$ and $\mathrm{H}_{2} 2.122 \mu \mathrm{m}$, to probe both the atomic and the molecular jet components.

Results. In the inner jet region of $\mathrm{HH} 34$, we find that both the atomic and molecular gas present two components at high and low velocity (the so-called HVC and LVC), as reported by previous studies. The [Fe II] LVC in HH34 is detected up to large distances from the source (>1000 AU), at variance with TTauri jets where the LVC is usually confined within $200 \mathrm{AU}$ from the star. In $\mathrm{H}_{2} 2.122 \mu \mathrm{m}$, the LVC and HVC are spatially separated, with an abrupt transition from low- to high-velocity emission at $\sim 11^{\prime \prime} 5$. We moreover detect, for the first time, the fainter red-shifted counterpart down to the central source. This lobe shows several emission knots displaced symmetrically with respect to the corresponding blue-shifted gas. In HH1, we trace the jet down to 1" from the VLA1 driving source: the kinematics of this inner region is again characterised by the presence of two velocity components, one blue-shifted and one red-shifted with respect to the source LSR velocity. We interpret this double component as arising from the interaction of two different jets. We suggest that the red-shifted component could be part of the HH501 jet. Electron densities and mass fluxes have been measured separately for the different velocity components in the HH34 and HH1 jets. In the inner HH34 jet region, $n_{\mathrm{e}}$ increases with decreasing velocity, with an average value of $\sim 1 \times 10^{4} \mathrm{~cm}^{-3}$ in the HVC and $\sim 2.2 \times 10^{4} \mathrm{~cm}^{-3}$ in the LVC. Up to $\sim 10^{\prime \prime}$ from the driving source, and along the whole HH1 jet an opposite behaviour is observed instead, with $n_{\mathrm{e}}$ increasing with velocity. In both jets the mass flux is carried mainly by the high-velocity gas: lower limits on the mass flux of $3-8 \times 10^{-8} M_{\odot} \mathrm{yr}^{-1}$ have been found for $\mathrm{HH} 34$ and $\mathrm{HH} 1$, from the luminosity of the [Fe II] $1.644 \mu \mathrm{m}$ line. A comparison between the position velocity diagrams and derived electron densities with models for magnetohydrodynamic (MHD) jet launching mechanisms has been performed for HH34. While the kinematical characteristics of the line emission at the jet base can be, at least qualitatively, reproduced by both $\mathrm{X}$-winds and disc-wind models, none of these models can explain the extent of the LVC and the velocity dependence of electron density that we observe. It is possible that the LVC in HH34 represents gas not directly ejected in the jet but instead denser ambient gas entrained by the high-velocity collimated jet.

Key words. stars: circumstellar matter, interstellar medium (ISM) - ISM: Herbig-Haro objects - ISM: jets and outflows ISM: individual objects: HH34 - ISM: individual objects: HH1

\section{Introduction}

Protostellar jets and accretion discs are intimately connected phenomena. The relation between both structures and the jet launching mechanism is, however, still not well understood. Several models have been developed to constrain the physical mechanism by which mass is accelerated from the star's vicinity (Shu et al. 1995; Ferreira 1997; Matt \& Pudritz 2005) by the action of so-called "rotating magnetospheres" (Camenzind 1990). Observations of the inner part of the jet structure are required, however, to verify the predictions of such models. In particular, near-infrared (NIR) spectroscopy is an important tool to investigate the jet structure closest to the driving source. This is especially true for jets in Class 0/I objects, where high extinction prevents us from observing the regions close to the source with standard optical tracers. Several observational studies have

* Based on observations collected at the European Southern Observatory, La Silla, Chile (ESO programmes 0.74.C-0286(A)). indeed been carried out during recent years employing near-IR line diagnostics on jet beams from young embedded sources (e.g., Nisini et al. 2005; Davis et al. 2006; Podio et al. 2006; Takami et al. 2006). In these studies, the main lines investigated are [Fe II] lines (e.g., $1.644 \mu \mathrm{m}$ and $1.600 \mu \mathrm{m}$ ) and $\mathrm{H}_{2}$ lines (the $2.12 \mu \mathrm{m}$ line is among the brightest lines). These works have addressed both the physical parameters and the kinematical properties of the sources, and their similarities with more evolved classical T Tauri stars (CTTSs). Kinematically, both Class I and CTT stars, show similar behaviour, with the presence of a collimated and large scale jet at high velocity $\left(\sim 200-400 \mathrm{~km} \mathrm{~s}^{-1}\right)$, called the high-velocity component (HVC), and a compact gas component localised around the central source at lower velocity $\left(\sim 0-50 \mathrm{~km} \mathrm{~s}^{-1}\right)$ named low-velocity component (LVC). This supports the assumption that the accretion and ejection in Class I objects occurs with the same mechanism as T Tauri stars. Synthetic position velocity (PV) diagrams constructed for different classes of magnetohydrodynamic (MHD) jet models (i.e., 
disc-winds, Garcia et al. 2001; and X-winds, Shang et al. 2002) predict the presence of different velocity components at the jet base, although details of the observed kinematical features are still not well reproduced by these models.

Concerning their physical properties, however, jets from Class I and CTTSs show some differences: in general, densities and mass fluxes derived for jets from Class I sources are higher than those in jets from CTTSs, as one would expect in sources with higher accretion rates embedded in a dense environment (e.g., Davis et al. 2003; Antoniucci et al. 2008). Moreover, the molecular component traced by $\mathrm{H}_{2}$ NIR lines is significantly enhanced in the younger sources, and in some of them the jet shows up only in molecular form.

In this framework, an important observational test is to measure the relevant physical parameters separately in the different velocity components of the flows. These diagnostics can show wether the differences in the physical properties between jets from Class I sources and CTTSs persist in both the HVC and LVC, and provide further constraints on the origin of both components. Differences in the excitation conditions of the various velocity components have been measured in jets from CTTSs. This kind of study has, however, never been attempted for jets from Class 0/I stars.

Here, we present recent results on the HH34 and HH1 jets, two classical outflows that are bright in the near-IR (e.g. Davis et al. 2000, 2001; Eislöffel et al. 2000). Their driving sources are HH34 IRS and VLA1, two young embedded Class 0/I objects situated at a distance of $450 \mathrm{pc}$ and $460 \mathrm{pc}$, respectively, in a high phase of accretion (Antoniucci et al. 2008; Chini et al. 2001). Velocity resolved, near-IR spectroscopy of HH34, addressing the kinematics of the inner region, has been presented by Davis et al. (2001), Davis et al. (2003) and Takami et al. (2006). More recently, we observed the HH34 and HH1 jets in low-resolution spectroscopy from 0.6 to $2.4 \mu \mathrm{m}$, and derived the relevant physical parameters of the jets, as a function of the distance from the exciting source, adopting an analysis combining optical and near-IR line ratios (Nisini et al. 2005; Podio et al. 2006). In this work, we present velocity-resolved NIR spectra obtained with the spectrograph ISAAC on the Very Large Telescope (VLT) of the European Southern Observatory (ESO). This instrument covers important diagnostic lines of the ionised ([Fe II] 1.644, $1.600 \mu \mathrm{m})$ and molecular $\left(\mathrm{H}_{2} 2.122 \mu \mathrm{m}\right)$ gas. We aim to study the kinematics of the atomic and molecular components, and to derive the electron density and mass ejection rates of the different velocity components. The paper is structured as follows: the observations and data reduction methods are described in Sect. 2. In Sect. 3, we present the results on the kinematics of the [Fe II] and $\mathrm{H}_{2}$ lines. In Sect. 4, we derive the electron density and mass flux from the [Fe II] line luminosity and ratios, and discuss the variation of these parameters as a function of velocity and distance from the central object. In Sect. 5, we discuss the comparison of our results with the predictions of proposed jet launching models. Finally, we draw our conclusions in Sect. 6.

\section{Observations and data reduction}

Observations were obtained on 28, 29 December 2004 at the ESO VLT telescope on Cerro Paranal, Chile, using the infrared spectrograph and camera ISAAC at medium resolution. The adopted slit width is 0.3 , corresponding to a nominal resolution $R \sim 8900$ in the $K$-band and 10000 in the $H$-band. The spatial scale of the camera is $0.146 /$ pixel. We have taken $H$ $(1.57-1.65 \mu \mathrm{m})$ and $K(2.07-2.20 \mu \mathrm{m})$ band spectral segments with the slit aligned along the jets. An additional segment in
$K$-band, covering the range $2.19-2.31 \mu \mathrm{m}$, was also acquired for $\mathrm{HH} 34$. The adopted position angles (PA) were $145^{\circ}$ and $-15^{\circ}$ for the $\mathrm{HH} 1$ and $\mathrm{HH} 34$ jets, respectively. Total integration times were $3300 \mathrm{~s}$ in the $K$-band for both the jets and $5100 \mathrm{~s}$ and $6900 \mathrm{~s}$ in the $H$-band for $\mathrm{HH} 1$ and $\mathrm{HH} 34$, respectively. We performed data reduction using standard $\mathrm{IRAF}^{1}$ tasks. The IR spectra were corrected for atmospheric spectral response by dividing the object spectra by the spectrum of a B5III star showing telluric lines. Wavelength calibration was performed using the atmospheric $\mathrm{OH}$ emission lines. The $H$ and $K$-band effective resolution measured from the $\mathrm{OH}$ lines is $\sim 8600$ and $\sim 7800$ for both objects. Flux calibration was performed using a photometric standard star. The standard stars have been observed at similar air mass and seeing conditions as the objects, therefore we applied no corrections for flux losses due to the slit width smaller than the seeing ( $\sim$.' 6 in both the nights). This procedure probably leads to some flux losses in the more external section of the jet $\left(\gtrsim 15^{\prime \prime}\right.$ from the source) where the intrinsic jet diameter, as measured from Hubble Space Telescope (HST) images (Reipurth et al. 2002) widens to more than $0^{\prime} 6$. In the case of HH34, we subtracted the continuum emission from the HH34 IRS source using the IRAF task BACKGROUND.

In the $H$-band spectral segments, only the [Fe II] 1.5999 and $1.6440 \mu \mathrm{m}$ lines have been detected, while several additional transitions have been observed in $K$-band. Figures 1 and 2 report the $K$-band spectral images showing the different emission lines found for the HH34 and HH1 jets, while the most prominent identified lines are listed in Table 1 for both jets. For HH34, we report here only the lines that are spatially resolved along the jet direction: additional transitions, mostly from permitted species, have been detected in the on-source spectrum and discussed in Antoniucci et al. (2008). Along the HH34 jet, our detected lines are consistent with those observed by Takami et al. (2006) except for our additional detection of the [Fe II] $2.224 \mu \mathrm{m}$ line, originating from the ${ }^{2} \mathrm{H}$ term. The [Fe II] $2.133 \mu \mathrm{m}$ line, connecting the ${ }^{2} \mathrm{P}$ and ${ }^{4} \mathrm{P}$ terms, has been detected in both the HH34 and HH1 jets: as pointed out by Takami et al. (2006), this transition, with excitation energies in excess of $25000 \mathrm{~K}$, is very useful to probe high-excitation regions, such as those found at the jet base or in high-velocity shock interaction. In HH1, we also detected two transitions from [Ti II], characterised by excitation temperature of the order of $7000 \mathrm{~K}$. Finally, in knot H of HH1, weak emission from $\mathrm{Br} \gamma$ is detected, testifying for the high-excitation conditions in this knot.

\section{3. [ $\mathrm{Fe} I I]$ and $\mathrm{H}_{2}$ kinematics}

In order to study the kinematics of the two jets in both the atomic and molecular components, we have constructed position-velocity (PV) diagrams of the [Fe II] $1.644 \mu \mathrm{m}$ and $\mathrm{H}_{2} \quad 2.122 \mu \mathrm{m}$ emission lines. The velocity is expressed with respect to the local standard of rest for both PV diagrams. A parental cloud velocity of $8 \mathrm{~km} \mathrm{~s}^{-1}$ and $10.6 \mathrm{~km} \mathrm{~s}^{-1}$ has been adopted for HH34 and HH1, respectively (Anglada et al. 1995; Choi \& Zhou 1997), and subtracted in the final PV velocity scale. Distance scales (in $\operatorname{arcsec}$ ) have been measured with respect to HH34 IRS and VLA1 for HH34 and HH1. Since the driving source VLA1 of the HH1 jet is not visible in the near-IR, we have used the bright Cohen-Schwartz (CS) star as the positional

\footnotetext{
1 IRAF (Image Reduction and Analysis Facility) is distributed by the National Optical Astronomy Observatories, which are operated by AURA, Inc., cooperative agreement with the National Science Foundation.
} 

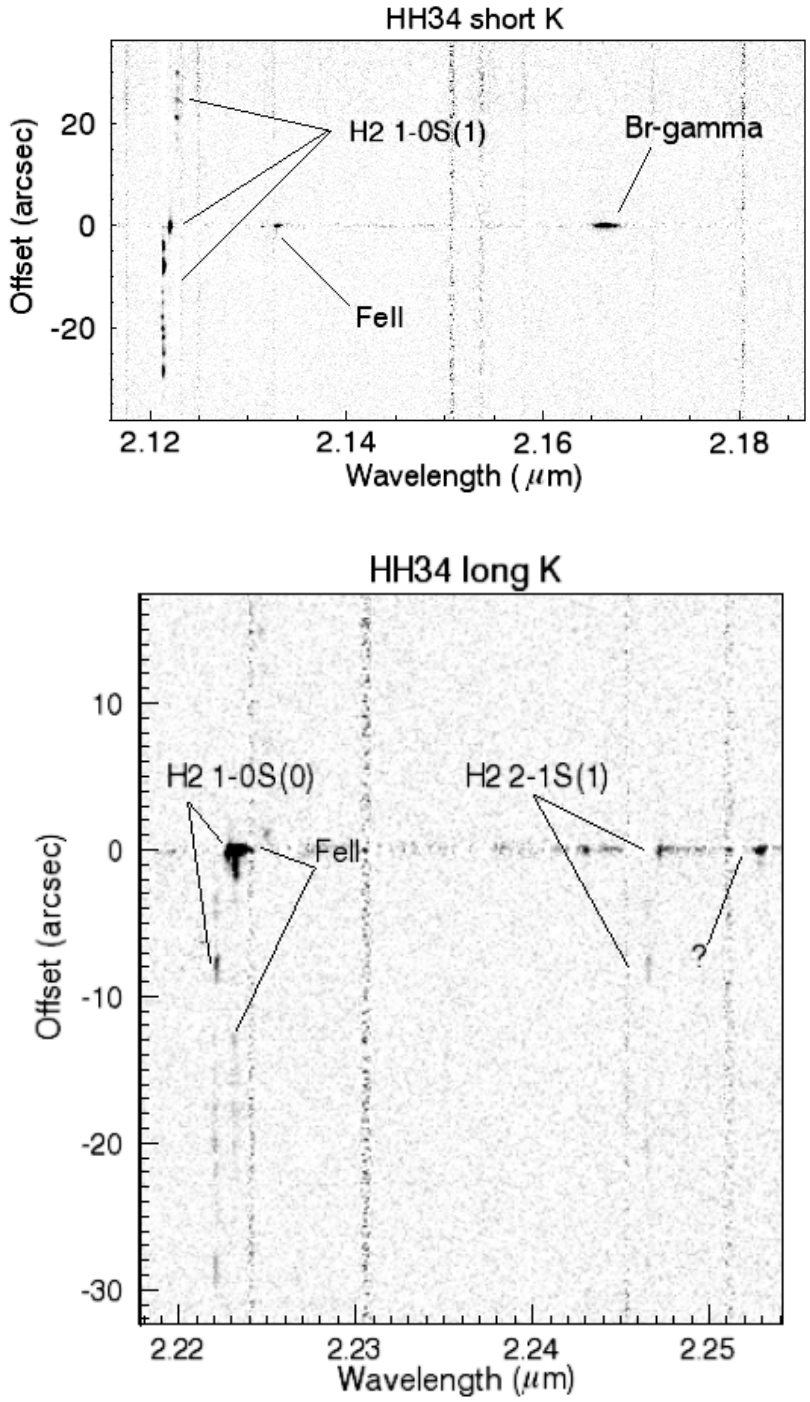

Fig. 1. Continuum-subtracted $K$-band spectral images of the HH34 jet.

reference, adopting for it an angular separation of 35.9 from the source VLA1. In the following, we will describe the results obtained for the two sources separately.

\section{1. $\mathrm{HH} 34$}

\subsubsection{Large scale properties}

Figures 3 and 4 show the [Fe II] $1.644 \mu \mathrm{m}$ and $\mathrm{H}_{2} 2.122 \mu \mathrm{m}$ lines PV diagrams relative to the blue-shifted and red-shifted lobes of HH34. The intensity scale is different in the two figures as evidenced by the relatively weak red-shifted emission. The redshifted counterpart of HH34 jet is clearly detected in our spectral images. We have named the detected blue-shifted emission knots from A to L, following the nomenclature of Eislöffel \& Mundt (1992) and Reipurth et al. (2002). We do not detect emission, however, from the knot B, since this knot is not aligned with the main jet axis, as evidenced in Reipurth et al. (2002). The next knots we observe are the knots $C$ and D, which we grouped together as knot $\mathrm{C}$. The red-shifted knots, which are located at approximately symmetric positions with respect to the corresponding blue-shifted knot, are named here from rA to $\mathrm{rH}$.
We computed the values of the [Fe II] and $\mathrm{H}_{2} 1-0 \mathrm{~S}(1)$ peak velocities applying a Gaussian fit to the line profile of every blue and red-shifted knot emission, and listed them in Table 2. In the knots closer to the star, where we identified two velocity components, we measured their peak velocity separately considering a two-Gaussian fit. The [Fe II] radial velocities in the blue lobe cover a range from -92 to $-108 \mathrm{~km} \mathrm{~s}^{-1}$, which is consistent with the values measured by Takami et al. (2006) and Davis et al. (2003), who found a range in radial velocities for the blue lobe from -90 to $-100 \mathrm{~km} \mathrm{~s}^{-1}$, corrected for a cloud velocity of $8 \mathrm{~km} \mathrm{~s}^{-1}$.

From knots A6 to I the blueshifted radial velocity increases from $\sim-92$ to $\sim-100 \mathrm{~km} \mathrm{~s}^{-1}$, passing through a maximum at $\sim-108 \mathrm{~km} \mathrm{~s}^{-1}$, then decreases again down to $\sim-92 \mathrm{~km} \mathrm{~s}^{-1}$ at knot K. Finally, it increases in knot $\mathrm{L}$ to $\sim-98 \mathrm{~km} \mathrm{~s}^{-1}$. Errors in relative velocities are estimated to be of the order of $2 \mathrm{~km} \mathrm{~s}^{-1}$, since our wavelength calibration has an uncertainty of $0.1 \AA$.' The [Fe II] radial velocity along the red lobe shows a similar behaviour. The velocity roughly increases from the knot closest to the source, $\mathrm{rA}$, to the knot rB, from $\sim 130 \mathrm{~km} \mathrm{~s}^{-1}$ to $\sim 140 \mathrm{~km} \mathrm{~s}^{-1}$, then decreases down to a value of $\sim 96 \mathrm{~km} \mathrm{~s}^{-1}$. Table 2 reports the velocity dispersion of the [Fe II] emission, measured from the $F W H M$ of the Gaussian fit, deconvolved for the instrumental profile. In knots A6-A3-A1, where different velocity components are evident (see Sect. 3.1.2) the reported velocity dispersion refers to the brightest component at high velocity. Intrinsic line widths of the order of $35-40 \mathrm{~km} \mathrm{~s}^{-1}$ are observed all along the jet. Under the assumption that the line emission arises from unresolved shock working surfaces, we find, following Hartigan et al. (1987), that the shock velocity is roughly given by $V_{\mathrm{s}} \sim \Delta V(F W Z I) \sim 2 \times \Delta V(F W H M)$. This implies shock velocities of the order of $70-80 \mathrm{~km} \mathrm{~s}^{-1}$, thus much higher than the value of $\sim 30 \mathrm{~km} \mathrm{~s}^{-1}$ estimated by Hartigan et al. (1994) on the basis of the comparison of optical line ratios with shock models. Indeed, shocks with speeds as high as $80 \mathrm{~km} \mathrm{~s}^{-1}$ are expected to produce a strong ionisation, on the order of $x_{\mathrm{e}} \sim 0.3-0.4$ (Hartigan et al. 1994), while Podio et al. (2006) measured an average ionisation along the HH34 jet of only 0.04 . Therefore, it seems that the line widening is determined not only by the shock, but also by, e.g., a lateral expansion of the jet.

The $\mathrm{H}_{2}$ radial velocities have a range from -89 to $-110 \mathrm{~km} \mathrm{~s}^{-1}$ for the blue lobe and an average value of $\sim+115 \mathrm{~km} \mathrm{~s}^{-1}$ for the red lobe. The $\mathrm{H}_{2}$ line profile is resolved all along the jet, with $\Delta V$ values of the order of $10-30 \mathrm{~km} \mathrm{~s}^{-1}$.

Also, in $\mathrm{H}_{2}$ radial velocities show cyclic variations on small scales along the jet with an increase of roughly $20 \mathrm{~km} \mathrm{~s}^{-1}$ from knot A6 to knot $\mathrm{E}$ and a subsequent decrease of the same order at knot K. It is known that the HH34 jet presents velocity variability on large and small spatial scales. Raga \& Noriega-Crespo (1998) and Raga et al. (2002) have shown that to reproduce the velocity pattern observed at different epochs, a model of variable ejection velocity including three modes with different periods is needed. The fastest of these modes can be represented with a sinusoid having a period of $27 \mathrm{yrs}$ and an amplitude of $\sim 15 \mathrm{~km} \mathrm{~s}^{-1}$. This is roughly consistent with our observed velocity pattern, that is reproduced quite closely by both [Fe II] and $\mathrm{H}_{2}$ and by the red-shifted and blue-shifted gas, clearly indicating its origin from ejection velocity variability. The velocity pattern observed at large distance may, however, be biased by the change in the jet axis direction that occurs at $d \sim 5^{\prime \prime}$ from the source, coupled with the widening of the jet diameter (up to $\sim 0$ ' $^{\prime} 6$ at $\sim 20$ arcsec from the source, Reipurth et al. 2002). Adopting an instrumental slit of only 0.3 , part of the kinematical components of the jet may not be properly probed by our observations. 
HH1 Short K

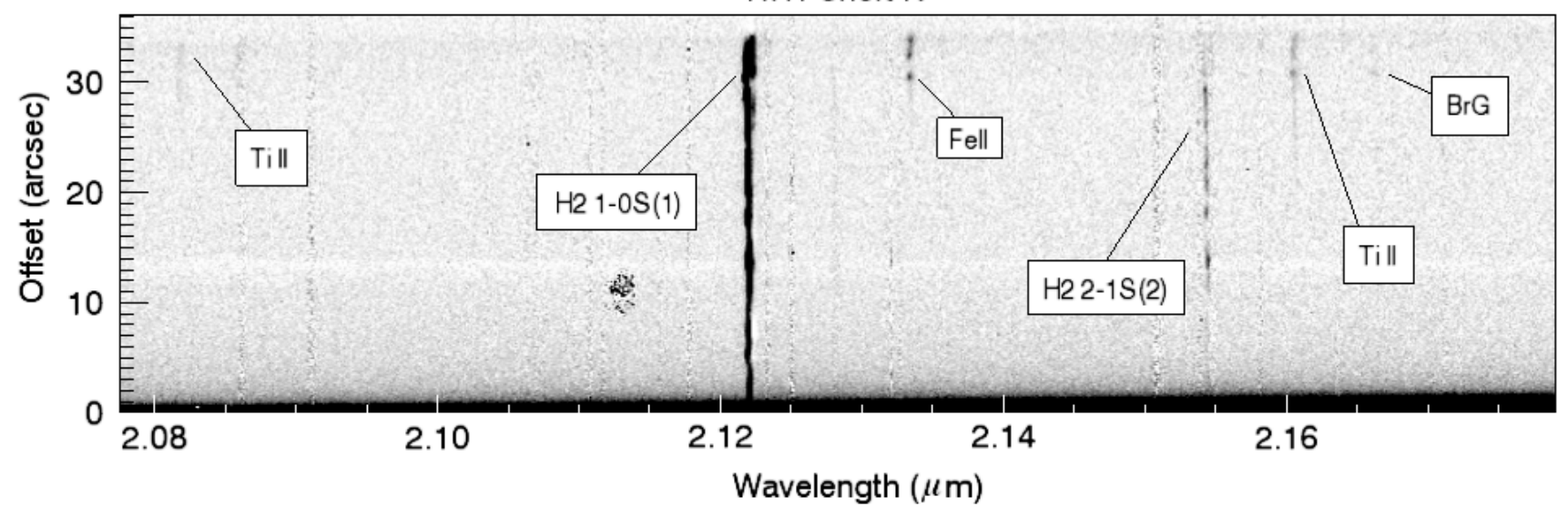

Fig. 2. Continuum-subtracted $K$-band spectral image of the HH1 jet.

Table 1. List of detected lines.

\begin{tabular}{ccc}
\hline \hline Line id. & $\lambda^{a}$ & Jet \\
\hline$[\mathrm{Fe} \mathrm{II}] a^{4} \mathrm{D}_{3 / 2}-a^{4} \mathrm{~F}_{7 / 2}$ & 1.5999 & $\mathrm{HH} 34, \mathrm{HH} 1$ \\
{$[\mathrm{Fe} \mathrm{II}] a^{4} \mathrm{D}_{7 / 2}-a^{4} \mathrm{~F}_{9 / 2}$} & 1.6440 & $\mathrm{HH} 34, \mathrm{HH} 1$ \\
{$[\mathrm{Fe} \mathrm{II}] a^{2} \mathrm{P}_{3 / 2}-a^{4} \mathrm{P}_{3 / 2}$} & 2.1333 & $\mathrm{HH} 34, \mathrm{HH} 1$ \\
{$[\mathrm{Fe} \mathrm{II}] a^{2} \mathrm{H}_{11 / 2}-a^{2} \mathrm{G}_{9 / 2}$} & 2.2244 & $\mathrm{HH} 34$ \\
{$[\mathrm{Ti} \mathrm{II}] a^{2} \mathrm{~F}_{5 / 2}-a^{4} \mathrm{~F}_{3 / 2}$} & 2.1605 & $\mathrm{HH} 1$ \\
{$[\mathrm{Ti} \mathrm{II}] a^{2} \mathrm{~F}_{7 / 2}-a^{4} \mathrm{~F}_{5 / 2}$} & 2.0818 & $\mathrm{HH} 1$ \\
$\mathrm{H}_{2} 1-0 \mathrm{~S}(1)$ & 2.1218 & $\mathrm{HH} 34, \mathrm{HH} 1$ \\
$\mathrm{H}_{2} 2-1 \mathrm{~S}(2)$ & 2.1542 & $\mathrm{HH} 1$ \\
$\mathrm{H}_{2} 1-0 \mathrm{~S}(0)$ & 2.2235 & $\mathrm{HH} 34$ \\
$\mathrm{H}_{2} 2-1 \mathrm{~S}(1)$ & 2.2477 & $\mathrm{HH} 34$ \\
$\mathrm{Br} \gamma$ & 2.1661 & $\mathrm{HH} 1$ \\
\hline
\end{tabular}

${ }^{a}$ Vacuum wavelengths in microns.

\subsubsection{Small scale properties}

Close to the central source, the [Fe II] lines broaden and emission at lower velocities, down to $0 \mathrm{~km} \mathrm{~s}^{-1}$, appears within $\sim 3^{\prime \prime}$ from the central source (Fig. 5). Inside $\sim 1^{\prime \prime}$ we see emission also at positive velocities, reconnecting with the spatially resolved red-shifted knot rA. This central region was already observed in [Fe II] and $\mathrm{H}_{2}$ by Davis et al. $(2001,2003)$ and Takami et al. (2006). Our observations have, however, a better spatial resolution than Davis et al. $(2001,2003)$ and are much deeper than those of Takami et al. (2006), who did not detect the [Fe II] redshifted component that we see at the jet base. We identify the high blue-shifted velocity corresponding to the large scale jet as the $\mathrm{HVC}$ and the emission component from 0 to $\sim 50 \mathrm{~km} \mathrm{~s}^{-1}$ LVC, in analogy with the HVC and LVC observed in the forbidden emission line (FEL) regions of $\mathrm{T}$ Tauri stars. We remark, however, that in T Tauri FELs one usually observes two separated peaks spatially located at different offsets from the central source, the HVC being commonly displaced further downstream (e.g. Hirth et al. 1997). Here the HVC has a peak on-source while the LVC is seen as a weaker shoulder of the brightest component. This behaviour could, however, be due to the lower resolution of our observations as a consequence of the larger distance of HH34 with respect to other studied T Tauri stars. When compared with other [Fe II] PV diagrams observed in T Tauri stars, HH34 is more similar to HL Tau and RW Aurigae (Pyo et al. 2006) than to L1551 and DG Tau (Pyo et al. 2002, 2003).

At variance with [Fe II], the $\mathrm{H}_{2} \mathrm{PV}$ diagram shows spatiallyand kinematically-separated LVC and HVC, and only the LVC
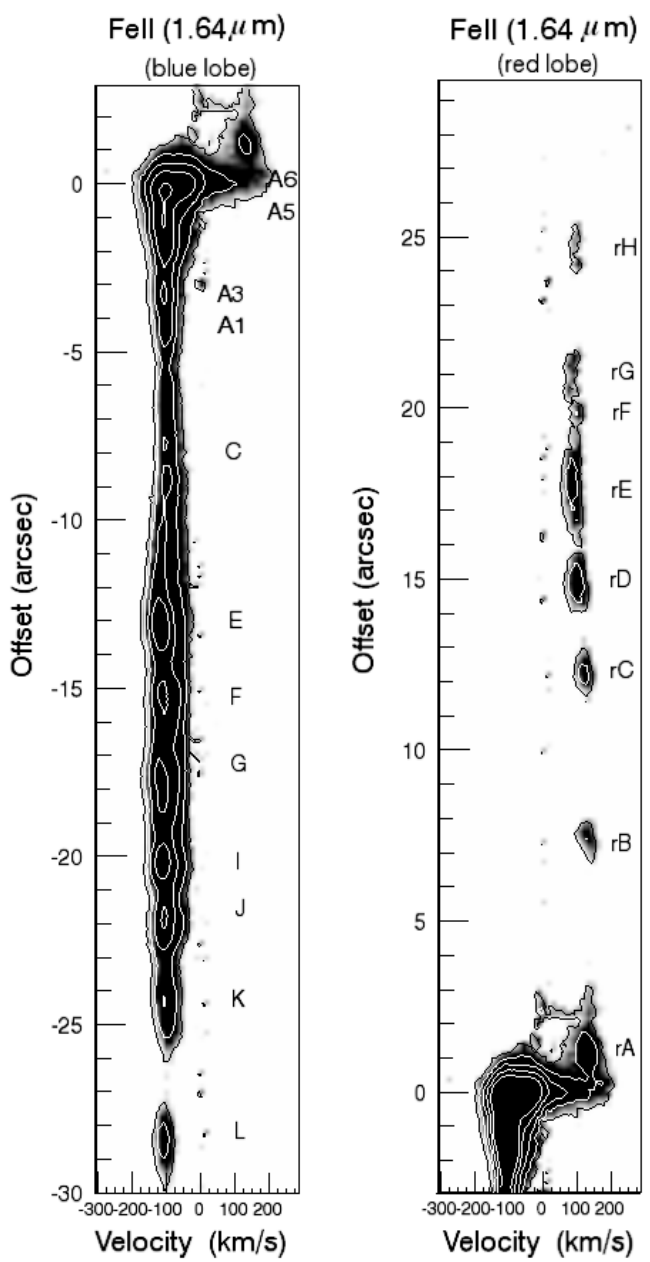

Fig. 3. Continuum-subtracted PV diagrams of the [Fe II] $1.644 \mu \mathrm{m}$ emission line for the blue and red lobe of the HH34 jet. A PA of $-15^{\circ}$ was adopted. Contours show values of 5, 15, 45, 135, 405 $\sigma$ for the blue lobe, and $5,10,20,40,80 \sigma$ for the red lobe. On the $Y$-axis the distance from HH34 IRS is reported.

is visible down to the central source. This component is close to $0 \mathrm{~km} \mathrm{~s}^{-1} \mathrm{LSR}$ velocity and the blue-shifted and red-shifted jets differ by less than $10 \mathrm{~km} \mathrm{~s}^{-1}$. The HVC appears at a distance of $2^{\prime \prime}$ from the central source, at the position of the knots A6 and rA. At intermediate velocities between these two 
Table 2. Observed radial velocities along the HH34 jet.

\begin{tabular}{|c|c|c|c|c|c|c|c|c|c|c|}
\hline \multicolumn{6}{|c|}{ Blue lobe } & \multicolumn{5}{|c|}{ Red lobe } \\
\hline \multirow[b]{2}{*}{ Knot } & \multirow[b]{2}{*}{$r_{\mathrm{t}}^{a}$} & \multicolumn{2}{|c|}{ [Fe II $] 1.64 \mu \mathrm{m}$} & \multicolumn{2}{|c|}{$\mathrm{H}_{2} 2.12 \mu \mathrm{m}$} & \multirow[b]{2}{*}{ Knot } & \multirow[b]{2}{*}{$r_{\mathrm{t}}^{a}$} & \multirow[t]{2}{*}[\mathrm{Fe}\mathrm{II}]{$1.64 \mu \mathrm{m}$} & \multicolumn{2}{|c|}{$\mathrm{H}_{2} 2.12 \mu \mathrm{m}$} \\
\hline & & $\mathrm{HVC}^{b}$ & $\mathrm{LVC}^{b}$ & $\mathrm{HVC}^{b}$ & $\mathrm{LVC}^{b}$ & & & & $\mathrm{HVC}^{b}$ & $\mathrm{LVC}^{b}$ \\
\hline A6 & 0.0 & $-92(46)$ & -52 & $-89(30)$ & -4 & $\mathrm{rA}$ & +1.0 & $+133(43)$ & & +10 \\
\hline A3 & -3.0 & $-98(37)$ & -67 & $-98(17)$ & -15 & $\mathrm{rB}$ & +7.3 & $+141(51)$ & & \\
\hline A1 & -4.5 & $-93(37)$ & -68 & $-95(12)$ & -7 & $\mathrm{rC}$ & +12.3 & $+128(58)$ & & \\
\hline $\mathrm{C}$ & -9.0 & $-92(43)$ & & $-94(12)$ & -7 & $\mathrm{rD}$ & +15.0 & $+108(35)$ & & \\
\hline E & -12.5 & $-108(44)$ & & $-110(8)$ & & $\mathrm{rE}$ & +17.4 & $+96(43)$ & & \\
\hline $\mathrm{F}$ & -15.2 & $-100(43)$ & & -107 (12) & & $\mathrm{rFG}$ & +21.0 & $+96(46)$ & $+113(26)$ & \\
\hline G & -17.2 & $-106(43)$ & & $-111(8)$ & & $\mathrm{rH}$ & +25.4 & $+100(35)$ & $+115(21)$ & \\
\hline I & -20.0 & $-100(43)$ & & $-105(24)$ & & & & & & \\
\hline $\mathrm{J}$ & -22.1 & $-96(43)$ & & -99 (19) & & & & & & \\
\hline K & -24.8 & $-92(44)$ & & $-92(26)$ & & & & & & \\
\hline $\mathrm{L}$ & -28.9 & $-98(26)$ & & -99 (19) & & & & & & \\
\hline
\end{tabular}

${ }^{a}$ Distance from the source in arcsec given by the mean value in the adopted aperture. Negative values correspond to the southeastern, blue-shifted jet axis.

${ }^{b}$ Radial velocities (in $\mathrm{km} \mathrm{s}^{-1}$ ) with respect to the local standard of rest and corrected for a cloud velocity of $8 \mathrm{~km} \mathrm{~s}^{-1}$. The radial velocity error is $2 \mathrm{~km} \mathrm{~s}^{-1}$. The velocity dispersion (in $\mathrm{km} \mathrm{s}^{-1}$ ), measured from the line $F W H M$ deconvolved for the instrumental profile, is reported in brackets for the HVC.
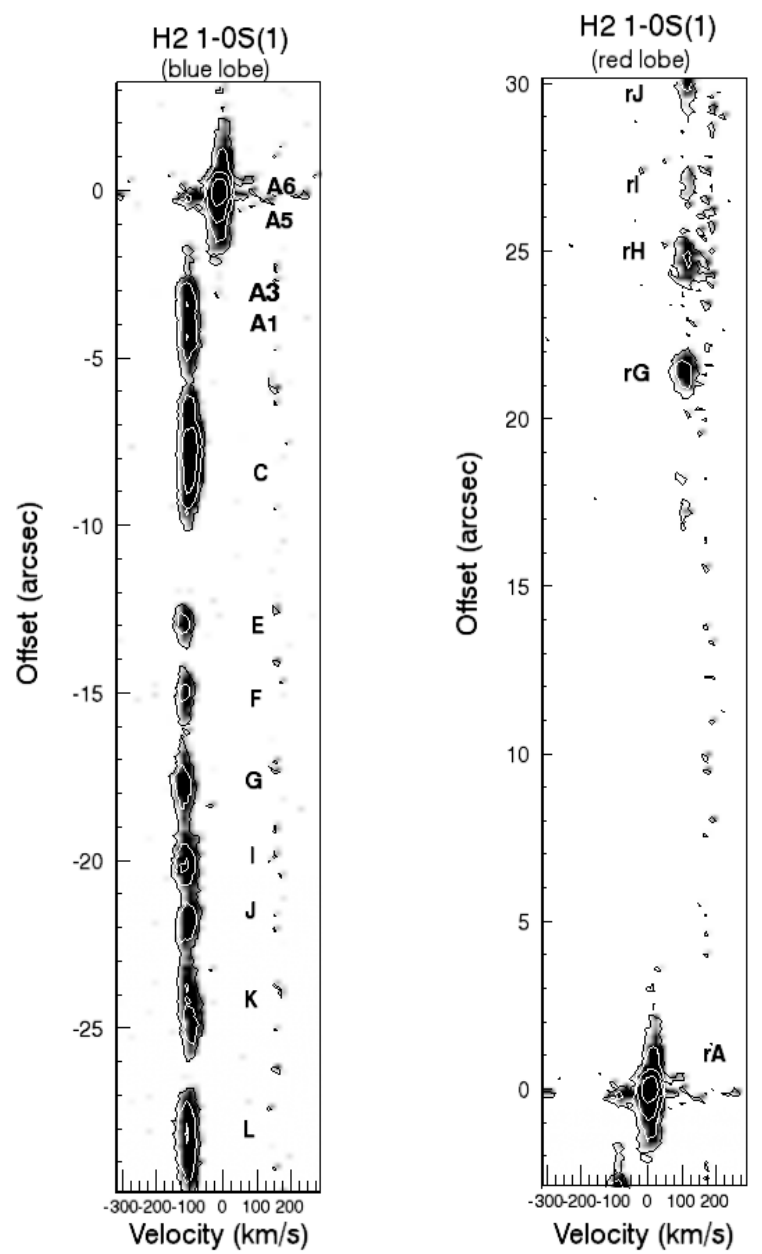

Fig. 4. Continuum-subtracted PV diagram for the $\mathrm{H}_{2} 1-0 \mathrm{~S}(1)$ emission line for the blue and red lobe of the HH34 jet. A PA of $-15^{\circ}$ was adopted. Contours show values of $5,15,45,135,405 \sigma$ for both the lobes. On the $Y$-axis the distance from HH34 IRS is reported.

components, no emission is seen in the $\mathrm{H}_{2} \mathrm{PV}$ diagram. This suggests that the two components correspond to physically distinct regions. The origin of the $\mathrm{H}_{2}$ high and low VC will be further discussed in Sect. 5.1.

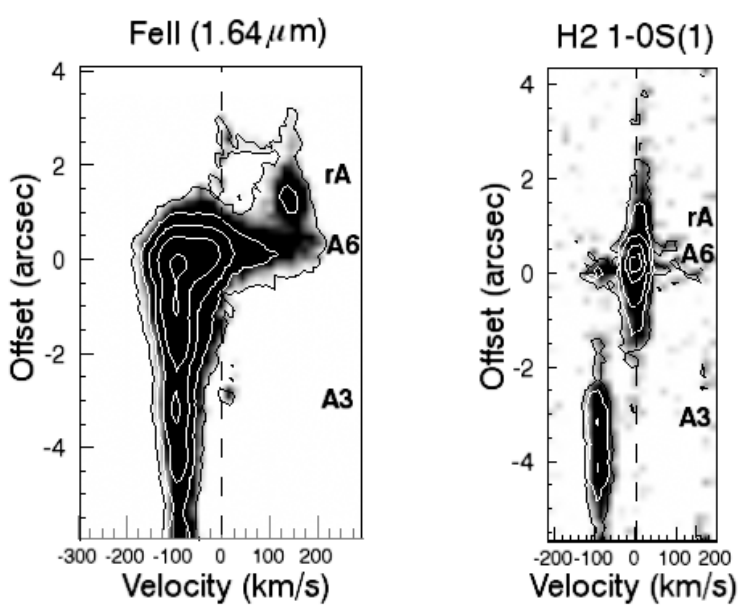

Fig. 5. Continuum-subtracted PV diagrams for the [Fe II] $1.644 \mu \mathrm{m}$ and $\mathrm{H}_{2}$ 1-0S(1) emission lines of the HH34 jet in the region nearest to the source. A PA of $-15^{\circ}$ was adopted. Contours show values of $5,15,45$, $135,260 \sigma$ for both lines. On the $Y$-axis the distance from HH34 IRS is reported.

\subsection{The HH1 jet}

Figures 6 and 7 show the PV diagrams of the $\mathrm{H}_{2} 2.122 \mu \mathrm{m}$ and $[\mathrm{Fe} \mathrm{II}] 1.644 \mu \mathrm{m}$ lines of the $\mathrm{HH} 1$ jet. The PVDs of the [Fe II] $2.133 \mu \mathrm{m}$ and [Ti II] $2.160 \mu \mathrm{m}$ lines are also presented in Fig. 7. The observed knots are named from KL to P, following the nomenclature by Eislöffel et al. (1994) and Bally et al. (2002). [Fe II] is detected only in the knots closer to the star, from KL to E, while $\mathrm{H}_{2}$ can be traced all along the jet; as already shown by Davis et al. (2000) and Nisini et al. (2005), the ratio $[\mathrm{Fe} \mathrm{II}] / \mathrm{H}_{2}$ sharply decreases with the distance from VLA1. Such a decrease is accompanied by a decrease of the $\mathrm{H}_{2}$ and [Fe II] radial velocities. Also the velocity dispersion diminishes from the internal to the external knots, as shown in Table 3. In particular, we measure intrinsic $F W H M$ (i.e., deconvolved by the instrumental width) decreasing from $\sim 64 \mathrm{~km} \mathrm{~s}^{-1}$ in knots KL to $\sim 20 \mathrm{~km} \mathrm{~s}^{-1}$ in knots G-F. As in HH34, these values imply high shock speeds (from $\sim 120 \mathrm{~km} \mathrm{~s}^{-1}$ in knot KL to $\sim 40 \mathrm{~km} \mathrm{~s}^{-1}$ in knot G), progressively decreasing outwards. Indications of shock velocities in the $\mathrm{HH} 1$ jet larger than in $\mathrm{HH} 34$ for the internal 


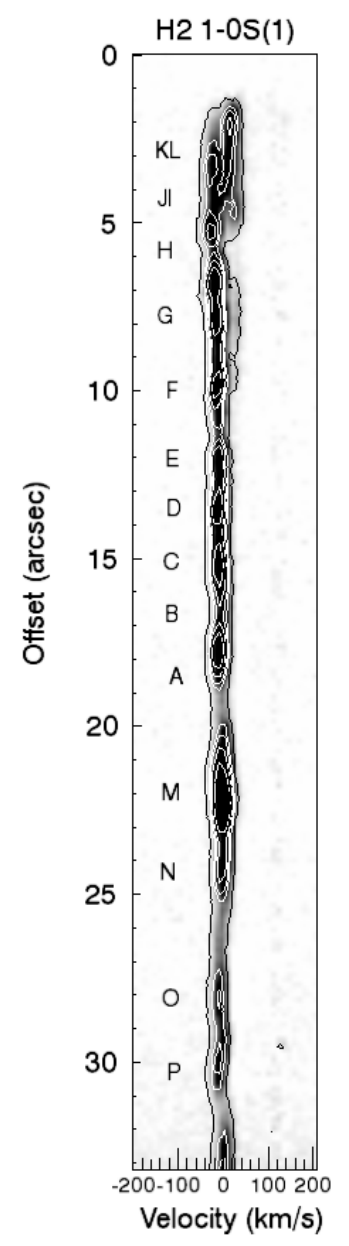

Fig. 6. Continuum-subtracted PV diagram for the $\mathrm{H}_{2}$ 1-0 $\mathrm{S}(1)$ line along the $\mathrm{HH} 1$ jet. A position angle of $145^{\circ}$ was adopted. Contours show values of $10,40,60,100 \sigma$. On the $Y$-axis the distance from the driving source VLA1 is reported.

knots are given by the detection of the $\mathrm{Br} \gamma$ and [Fe II] $2.133 \mu \mathrm{m}$ lines in knot $\mathrm{H}$. These lines are not detected in knots KL and JI probably due to the larger extinction. The [Fe II] and $\mathrm{H}_{2}$ radial velocities appear double-peaked from knot $\mathrm{KL}$ to $\mathrm{H}$ in [Fe II] and from knot $\mathrm{KL}$ to $\mathrm{F}$ in $\mathrm{H}_{2}$. Both, [Fe II] and $\mathrm{H}_{2}$ present one of the two components red-shifted with respect to the LSR.

The $[\mathrm{Fe}$ II] blue-shifted radial velocity increases from knot KL to knot JI from a value of $\sim-28 \mathrm{~km} \mathrm{~s}^{-1}$ to $\sim-46 \mathrm{~km} \mathrm{~s}^{-1}$ and then decreases again to a velocity of $\sim-24 \mathrm{~km} \mathrm{~s}^{-1}$ in knot $\mathrm{E}$. The red-shifted component is, however, too faint to be fitted with a Gaussian profile. Nevertheless, we can estimate an average value of $\sim+30 \mathrm{~km} \mathrm{~s}^{-1}$ from knot KL to $\mathrm{H}$.

On the other hand, in the $\mathrm{H}_{2}$ blue-shifted component the velocity increases from knot KL to knot JI, as in the case of [Fe II] and then roughly decreases to $\sim-3 \mathrm{~km} \mathrm{~s}^{-1}$ in knot A. After that, the radial velocity increases again to a value of around $-12 \mathrm{~km} \mathrm{~s}^{-1}$ in knot $\mathrm{N}$. The red-shifted component covers a velocity range from +6 to $+23 \mathrm{~km} \mathrm{~s}^{-1}$. The $\mathrm{H}_{2}$ line profiles are represented in Fig. 8 for several knots in the region closest to the source. The measured $\mathrm{H}_{2}$ velocities are consistent with the velocities reported in Davis et al. (2000) for the knots F and A. The $\mathrm{H}_{2}$ lines are non-resolved implying an intrinsic line width less than $39 \mathrm{~km} \mathrm{~s}^{-1}$. The origin of the double velocity component can be due either to the presence of another jet (e.g., the one driving the HH501 object Reipurth et al. 2000; Bally et al. 2002) or to the emission along the wings of a bow shock seen
Table 3. Observed radial velocities along the HH1 jet.

\begin{tabular}{cc|c|cc}
\hline \hline Knot & $r_{\mathrm{t}}{ }^{a}$ & {$[\mathrm{Fe}$ II $] 1.64 \mu \mathrm{m}$} & \multicolumn{2}{|c}{$\mathrm{H}_{2} 2.12 \mu \mathrm{m}$} \\
& $\left.V^{b}{ }^{b}\right)$ & \multicolumn{2}{|c}{$V_{\mathrm{r}}^{b}$} \\
& & & Blue & Red \\
\hline KL & 1.6 & $-28(64)$ & -16 & +13 \\
$\mathrm{JI}$ & 3.4 & $-46(53)$ & -27 & +6 \\
$\mathrm{H}$ & 5.1 & $-43(47)$ & -24 & +14 \\
$\mathrm{G}$ & 7.5 & $-35(22)$ & -17 & +23 \\
$\mathrm{~F}$ & 9.8 & $-31(20)$ & -14 & +17 \\
$\mathrm{E}$ & 11.6 & $-24(29)$ & -11 & \\
$\mathrm{D}$ & 13.6 & & -12 & \\
$\mathrm{C}$ & 15.0 & & -10 & \\
$\mathrm{~B}$ & 17.0 & & -11 & \\
$\mathrm{~A}$ & 18.2 & & -3 & \\
$\mathrm{M}$ & 22.1 & & -4 & \\
$\mathrm{~N}$ & 23.5 & & -12 & \\
\hline
\end{tabular}

${ }^{a}$ Distance from the source in arcsec given by the mean value in the adopted aperture.

${ }^{b}$ Radial velocities (in $\mathrm{km} \mathrm{s}^{-1}$ ) with respect to the LSR and corrected for a cloud velocity of $10.6 \mathrm{~km} \mathrm{~s}^{-1}$. The radial velocity error is $2 \mathrm{~km} \mathrm{~s}^{-1}$. The velocity dispersion (in $\mathrm{km} \mathrm{s}^{-1}$ ), measured from the line $F W H M$ deconvolved for the instrumental profile, is reported in brackets for the HVC.

almost perpendicular to the line of sight. These two possibilities will be further discussed in Sect. 4.1.2.

\section{Diagnostics of physical parameters}

\subsection{Electron density}

The electron density in the atomic jet component can be derived from the ratio of the [Fe II] 1.600/1.644 $\mu \mathrm{m}$ lines. This ratio is sensitive to $n_{\mathrm{e}}$ values between $\sim 10^{3}$ and $10^{5} \mathrm{~cm}^{-3}$, while it depends only weakly on the temperature (e.g. Nisini et al. 2002). Electron densities of the $[\mathrm{Fe}$ II] emission line region as a function of the distance from the driving source has been measured by Podio et al. (2006) and Nisini et al. (2005) for the HH34 and HH1 jets. In addition, Takami et al. (2006) provide the electron density in the inner region of HH34. Taking advantage of the velocity resolved profiles in both the 1.644 and $1.600 \mu \mathrm{m}$ lines, we can now measure the electron density in the different velocity components. To do that, we have extracted the spectra of the two lines at different positions along the flows and measured the $1.600 \mu \mathrm{m} / 1.644 \mu \mathrm{m}$ intensity ratio in each pixel along the spectral profile. Figures 9 and 10 show the normalised profiles of the two lines and their ratio as a function of velocity for HH34 and HH1. The spatial intervals used to extract the spectra of the individual knots are given in Tables 4 and 5. The [Fe II] line ratio has been computed only for the velocity points where the intensity in both the lines has been measured with a S/N larger than three. The plotted ratio gives a qualitative indication on how $n_{\mathrm{e}}$ varies in the different velocity components, that is, a higher ratio indicates a higher electron density. In the internal knots of $\mathrm{HH} 34$ (from A6 to A3) the $1.600 \mu \mathrm{m} / 1.644 \mu \mathrm{m}$ ratio decreases by $\sim 70 \%$ going from $\sim-50 \mathrm{~km} \mathrm{~s}^{-1}$ to $-100 \mathrm{~km} \mathrm{~s}^{-1}$. In the knots farther from the central source, on the other hand, the maximum $1.600 \mu \mathrm{m} / 1.644 \mu \mathrm{m}$ ratio is observed at the radial velocity peak, with some evidence that the ratio decreases in the line wings at both higher and lower velocities. In $\mathrm{HH} 1$, the $1.600 \mu \mathrm{m}$ line has been detected with a $\mathrm{S} / \mathrm{N}$ larger than three only in the internal knots, from JI to F. Here, the $1.600 \mu \mathrm{m} / 1.644 \mu \mathrm{m}$ ratio has a minimum close to $0 \mathrm{~km} \mathrm{~s}^{-1}$ velocity and increases, up to a factor of two in knot KL, toward high velocities. This behaviour is 

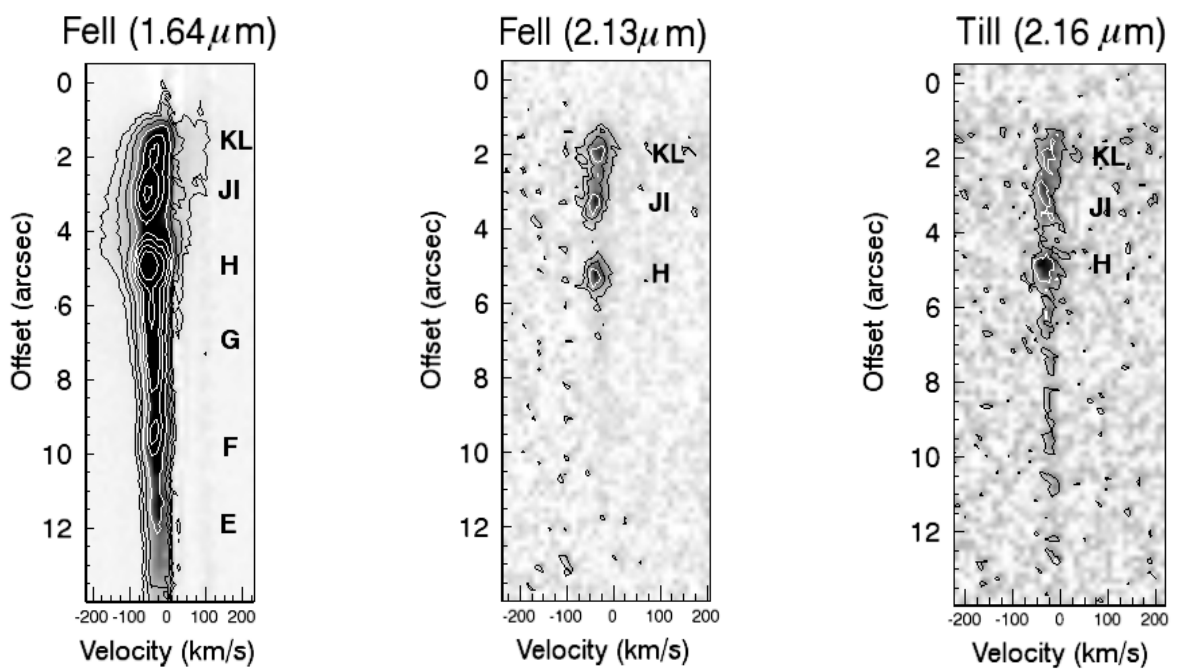

Fig. 7. PV diagrams for the [Fe II] $1.64 \mu \mathrm{m}, 2.13 \mu \mathrm{m}$ and [Ti II] $2.16 \mu \mathrm{m}$ emission lines of the HH1 jet. A position angle of $145^{\circ}$ was adopted. Contours show values of $11,22, \ldots, 176 \sigma ; 4,8,12 \sigma$ and $3,6,12 \sigma$, respectively. On the $Y$-axis the distance from the driving source VLA1 is reported.

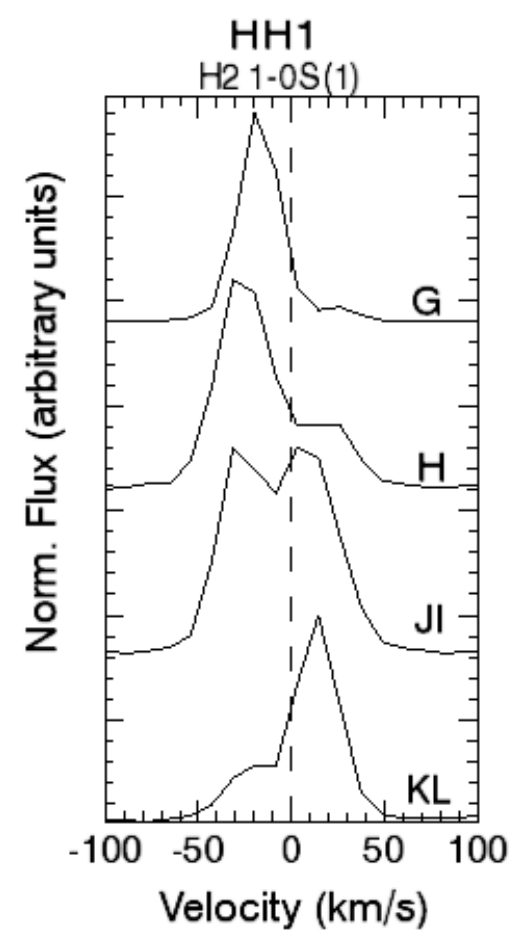

Fig. 8. Normalised line profiles of the $\mathrm{H}_{2}$ 1-0S(1) for different knots near the source.

therefore different from what has been observed in the internal knots of HH34. In HH1, however, the inner jet region is not detected due to the high extinction and therefore here we are not observing regions at the jet base, as we do in HH34. It seems indeed that the velocity dependence of the electron density is different between the FEL regions close to the star and the knots along the jet beam.

In order to have a quantitative determination of the electron density in the different velocity components, we have separated in the line profile the contribution from the HV and the LV components, and applied a 16 level $\mathrm{Fe}^{+}$statistical equilibrium model (Nisini et al. 2002). In the following, the results are discussed separately for the two sources.

\subsubsection{The $\mathrm{HH} 34$ jet}

In HH34, we have defined the HVC and LVC velocity ranges from the profile of the $1.600 \mu \mathrm{m}$ line of knot A6, where the two components have been fitted with a two-Gaussian fit. We have then measured the line ratios considering the intensities integrated in the FWHM ranges of these two Gaussians also for all the other knots. The considered velocity bins are from $\sim-120 \mathrm{~km} \mathrm{~s}^{-1}$ to $\sim-66 \mathrm{~km} \mathrm{~s}^{-1}$ (HV) and from $\sim-66 \mathrm{~km} \mathrm{~s}^{-1}$ to $\sim-7 \mathrm{~km} \mathrm{~s}^{-1}(\mathrm{LV})$. The adopted $A_{\mathrm{v}}$ value is given in Table 4, together with the derived electron densities in the two components. Figure 11 plots the derived values of $n_{\mathrm{e}}$ as a function of the distance from the source. The first trend that we notice is a sharp decrease of the HVC electron density (from $\sim 10^{4}$ to $\sim 2 \times$ $10^{3} \mathrm{~cm}^{-3}$ ) from the knot A6 to the other knots at distances farther than 2'. 5 from the source. Such a decrease in $n_{\mathrm{e}}$ has also been observed in Podio et al. (2006) and the values they derived, scaled for the different considered spatial regions observed through slits of slightly different width, are consistent with our measured values for $n_{\mathrm{e}}$. In the red-shifted knot rA, which is the only knot where significant $1.600 \mu \mathrm{m}$ emission has been detected in the red-lobe, we find a value for $n_{\mathrm{e}} \sim 1.2 \times 10^{4} \mathrm{~cm}^{-3}$, i.e., comparable to the value derived in knot A6. Secondly, as shown in Fig. 11, the values for $n_{\mathrm{e}}$ in the LVC are higher than in the HVC: in knot A6, the LVC electron density is $2.2 \times 10^{4} \mathrm{~cm}^{-3}$, i.e., $70 \%$ higher than in the HVC. About the same percentage is measured in knots A1 and A3. From the information in our data, we are unable to disentangle whether the larger electron density in the LVC with respect to the HVC is due to a higher total density or to a higher ionisation fraction. Our result is, however, in agreement with what is found in the FEL regions of T Tauri stars, where the emission component associated with the lowvelocity gas is denser and less excited than the HVC (Hamann et al. 1994; Hartigan et al. 1995). In such studies, the LVC was not spatially resolved and the different values for $n_{\mathrm{e}}$ found between the HVC and LVC were interpreted assuming that the LVC originates from a dense compact region close to the disc surface, while the HVC is associated with a more extended highvelocity jet displaced further out. Such an interpretation was supported by the spatial offset from the central source often found between the two components of the forbidden lines detected in spatially-unresolved spectra of T Tauri stars. Spatially-resolved 

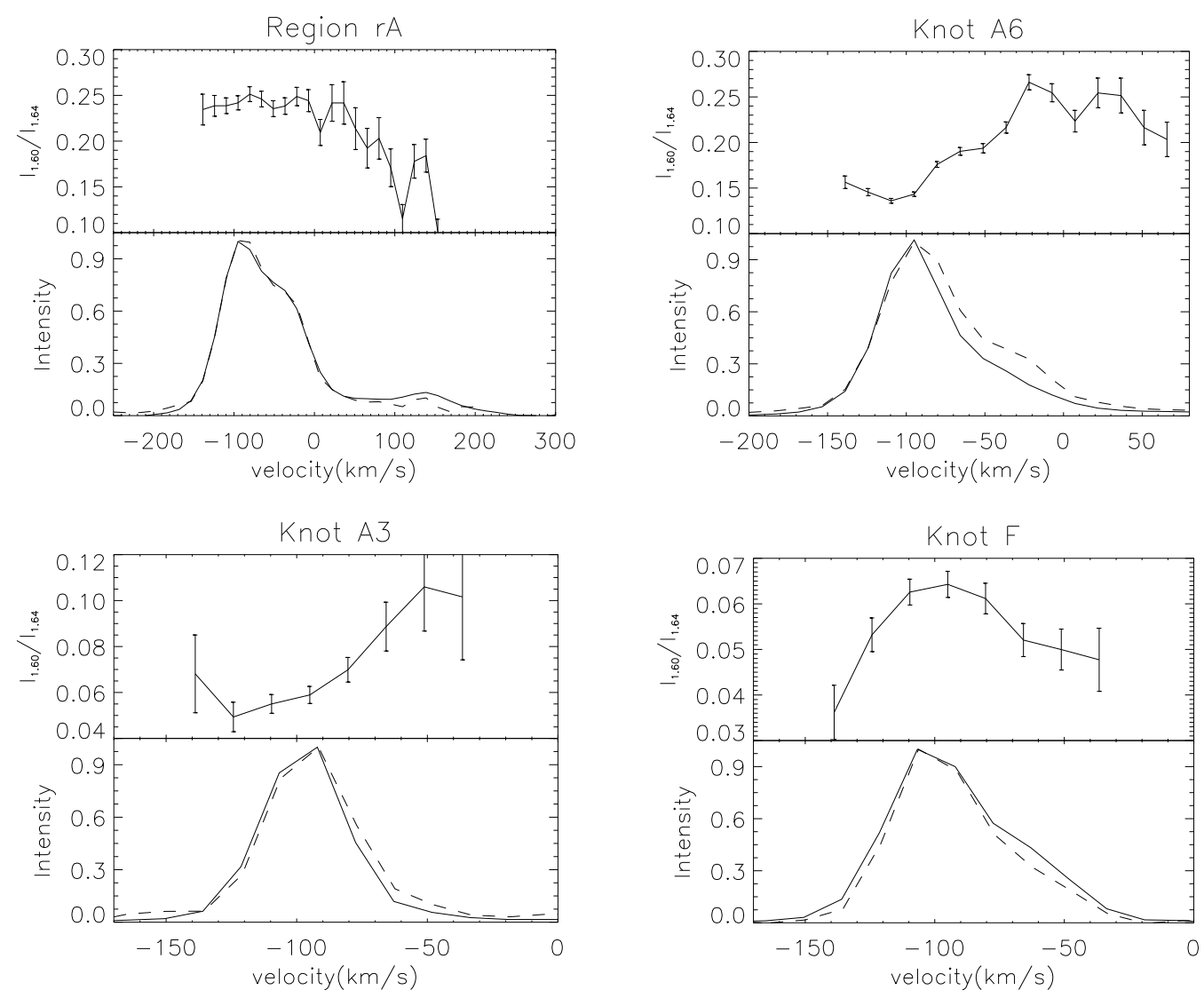

Fig. 9. Normalised line profiles (lower panels) of the [Fe II] lines $1.644 \mu \mathrm{m}$ (solid line) and $1.600 \mu \mathrm{m}$ (dotted line), and their ratio in each velocity channel (upper panel) for different extracted knots along the HH34 jet. Note that rA is a region that includes the knot rA.

Table 4. $\dot{M}_{\text {jet }}$ along the HH34 jet.

\begin{tabular}{|c|c|c|c|c|c|c|c|c|}
\hline Knot & $\begin{array}{l}r_{\mathrm{t}}^{a} \\
\left({ }^{\prime \prime}\right)\end{array}$ & $\begin{array}{l}A_{\mathrm{V}}^{b} \\
\mathrm{mag}\end{array}$ & $\begin{array}{c}V_{\mathrm{t}}(\mathrm{HVC})^{c} \\
\left(\mathrm{~km} \mathrm{~s}^{-1}\right)\end{array}$ & $\begin{array}{l}n_{\mathrm{e}}(\mathrm{HVC})^{d} \\
\left(10^{3} \mathrm{~cm}^{-3}\right)\end{array}$ & $\begin{array}{c}\dot{M}_{\mathrm{jet}}(\mathrm{HVC})^{e} \\
\left(M_{\odot} \mathrm{yr}^{-1}\right)\end{array}$ & $\begin{array}{c}V_{\mathrm{t}}(\mathrm{LVC})^{c} \\
\left(\mathrm{~km} \mathrm{~s}^{-1}\right)\end{array}$ & $\begin{array}{c}n_{\mathrm{e}}(\mathrm{LVC})^{d} \\
\left(10^{3} \mathrm{~cm}^{-3}\right)\end{array}$ & $\begin{array}{c}\dot{M}_{\text {jet }}(\mathrm{LVC})^{e} \\
\left(M_{\odot} \mathrm{yr}^{-1}\right)\end{array}$ \\
\hline A6 & $(-2.6,+0.9)$ & 7.1 & 218 & 10.5 & $5.2 \times 10^{-8}$ & 124 & 22.5 & $6.6 \times 10^{-9}$ \\
\hline A3 & $(-3.8,-2.6)$ & 7.1 & 232 & 1.8 & $8.3 \times 10^{-8}$ & 159 & 5.0 & $2.0 \times 10^{-9}$ \\
\hline A1 & $(-5.4,-3.8)$ & 7.1 & 220 & 1.8 & $3.3 \times 10^{-8}$ & 162 & 5.0 & $1.3 \times 10^{-9}$ \\
\hline $\mathrm{C}$ & $(-9.9,-8.0)$ & 7.1 & 218 & 1.2 & $4.4 \times 10^{-8}$ & & & \\
\hline E & $(-14.1,-11.0)$ & 1.3 & 255 & 1.8 & $5.9 \times 10^{-8}$ & & & \\
\hline $\mathrm{F}$ & $(-16.6,-14.1)$ & 1.3 & 236 & 1.8 & $5.0 \times 10^{-8}$ & & & \\
\hline $\mathrm{G}$ & $(-17.8,-16.6)$ & 1.3 & 251 & 2.5 & $1.0 \times 10^{-7}$ & & & \\
\hline I & $(-21.0,-19.2)$ & 1.3 & 236 & 2.5 & $4.2 \times 10^{-8}$ & & & \\
\hline $\mathrm{J}$ & $(-23.3,-21.0)$ & 1.3 & 227 & 1.2 & $3.9 \times 10^{-8}$ & & & \\
\hline $\mathrm{K}$ & $(-26.1,-23.5)$ & 1.3 & 217 & 1.5 & $1.0 \times 10^{-8}$ & & & \\
\hline $\mathrm{L}$ & $(-30.3,-27.5)$ & & 232 & & & & & \\
\hline $\mathrm{rA}$ & $(0,+2.0)$ & 7.1 & 218 & 12.0 & $2.2 \times 10^{-9}$ & & & \\
\hline
\end{tabular}

${ }^{a}$ Distance from the source in arcsec. Negative values correspond to the southeastern, blue-shifted jet axis, ${ }^{b}$ visual extinction from Podio et al. (2006), ${ }^{c}$ tangential velocity assuming an inclination of the jet $i=22.7$ to the plane of the sky (Eislöffel \& Mundt 1992), ${ }^{d}$ electron density for the $\mathrm{HV}$ and LV components, ${ }^{e} \dot{M}_{\text {jet }}$ for the $\mathrm{HV}$ and LV components assuming an electron temperature of $7000 \mathrm{~K}$.

measurements of the electron density in the HV and LV components have been performed in DG Tau by Bacciotti et al. (2000) and Lavalley-Fouquet et al. (2000). In this case, at variance with $\mathrm{HH} 34$, the electron density has been found to increase with velocity, up to a distance of $\sim 450 \mathrm{AU}$ from the central source. On the other hand, in the DG Tau micro-jet the total density in the LV component is higher than in the HV component because of the much lower ionisation fraction. Therefore, the density structure in HH34 and in the T Tauri stars might be similar, but they differ in the excitation conditions. In HH34, we spatially resolve the LVC, whose emission is traceable up to $\sim 5$ arcsec from the source, i.e., more than 2000 AU. Therefore, the spatial scale between the DG Tau micro-jet and the HH34 jet are very different: in addition, and at variance with many $\mathrm{T}$ Tauri stars, we do not observe a spatial offset between the HV and LV components, although this may be a result of the moderate spatial resolution of our observations. Therefore, it may be that the LVC that we are tracing with our observations has a different origin than in the CTTS case. In the outer knots, i.e., from $\mathrm{C}$ outwards, we cannot distinguish high and low velocity components anymore. 

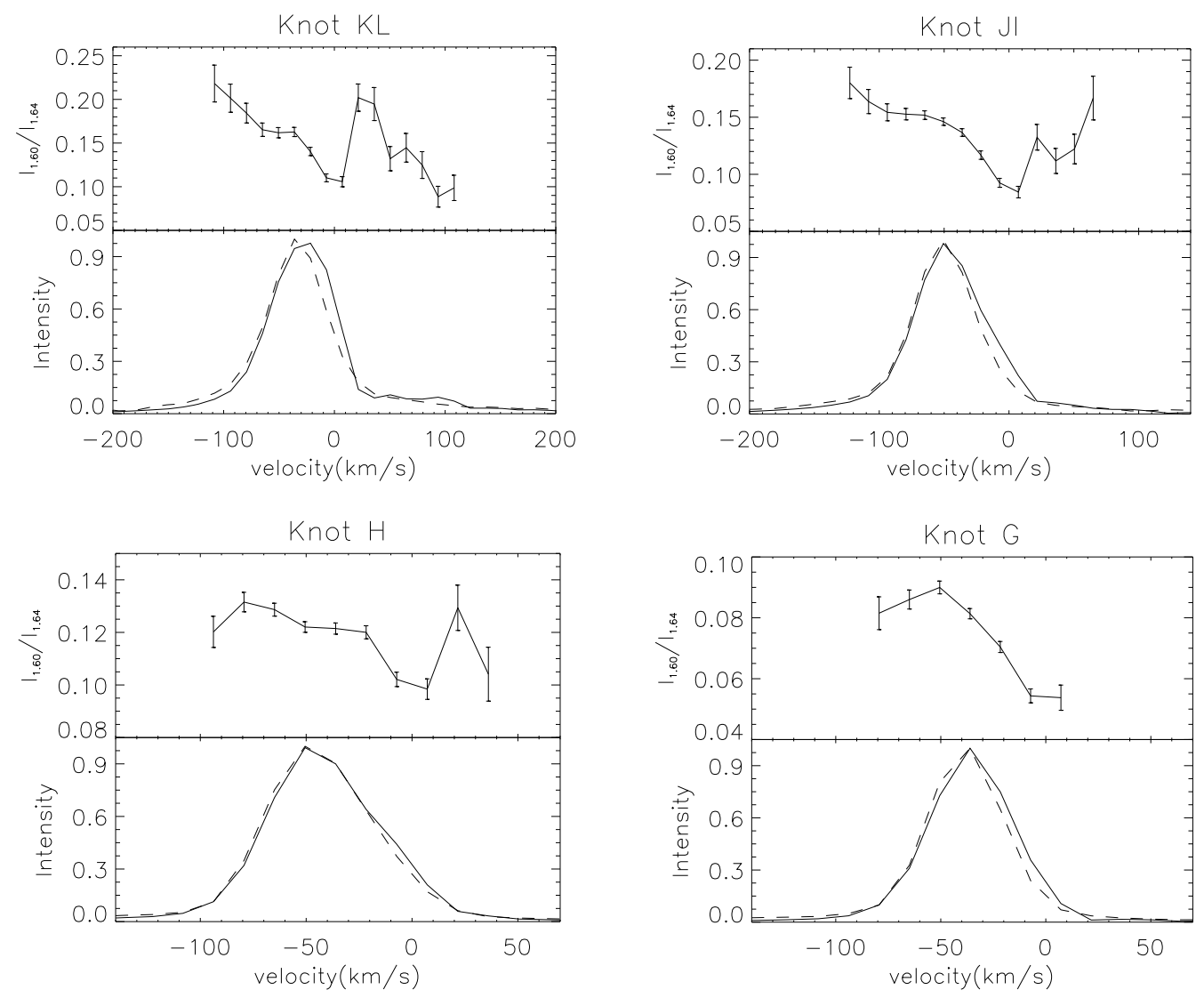

Fig. 10. Normalised line profiles (lower panels) of the [Fe II] lines $1.644 \mu \mathrm{m}$ (solid line) and $1.600 \mu \mathrm{m}$ (dotted line), and their ratio in each velocity channel (upper panel) for different extracted knots along the HH1 jet.

Table 5. $\dot{M}_{\text {jet }}$ along the HH1 jet.

\begin{tabular}{c|ccc|ccc|ccc}
\hline \hline Knot & $\begin{array}{c}r_{\mathrm{t}}{ }^{2} \\
\left({ }^{\prime}\right)\end{array}$ & $\begin{array}{c}A_{\mathrm{v}}{ }^{b} \\
\mathrm{mag}\end{array}$ & $\begin{array}{c}T_{\mathrm{e}}^{b} \\
\left(10^{3} \mathrm{~K}\right)\end{array}$ & $\begin{array}{c}V_{\mathrm{t}}(\mathrm{HVC})^{c} \\
\left(\mathrm{~km} \mathrm{~s}^{-1}\right)\end{array}$ & $\begin{array}{c}n_{\mathrm{e}}(\mathrm{HVC})^{d} \\
\left(10^{3} \mathrm{~cm}^{-3}\right)\end{array}$ & $\begin{array}{c}\dot{M}_{\mathrm{jet}}(\mathrm{HVC})^{e} \\
\left(M_{\odot} \mathrm{yr}^{-1}\right)\end{array}$ & $\begin{array}{c}V_{\mathrm{t}}(\mathrm{LVC})^{c} \\
\left(\mathrm{~km} \mathrm{~s}^{-1}\right)\end{array}$ & $\begin{array}{c}\left.n_{\mathrm{e}}(\mathrm{LVC})\right)^{d} \\
\left(10^{3} \mathrm{~cm}^{-3}\right)\end{array}$ & $\begin{array}{c}\dot{M}_{\mathrm{jet}}(\mathrm{LVC})^{e} \\
\left(M_{\odot} \mathrm{yr}^{-1}\right)\end{array}$ \\
\hline $\mathrm{KL}$ & $(0.7,2.6)$ & 8.3 & 11 & 159 & 10.6 & $1.3 \times 10^{-8}$ & 187 & 9.8 & $2.3 \times 10^{-9}$ \\
$\mathrm{JI}$ & $(2.6,4.3)$ & 8.3 & 11 & 261 & 9.0 & $3.6 \times 10^{-8}$ & 187 & 8.3 & $3.1 \times 10^{-9}$ \\
$\mathrm{H}$ & $(4.2,6.1)$ & 2.9 & 9.2 & 244 & 8.3 & $4.4 \times 10^{-8}$ & & & \\
$\mathrm{G}$ & $(6.1,8.9)$ & 2.0 & 10.5 & 198 & 4.6 & $2.7 \times 10^{-8}$ & & & \\
$\mathrm{~F}$ & $(8.9,10.7)$ & 2.0 & 9.8 & 176 & 3.8 & $2.2 \times 10^{-9}$ & & & \\
\hline
\end{tabular}

${ }^{a}$ Distance from VLA1 in arcsec, ${ }^{b}$ visual extinction and electron temperature from Nisini et al. (2005), ${ }^{c}$ tangential velocity assuming an inclination of the jet $i=10^{\circ}$ to the plane of the sky (Bally et al. 2002), ${ }^{d}$ electron density for the HV and LV components, ${ }^{e} \dot{M}_{\text {jet }}$ for the HV and LV components.

Instead, the density here seems to have the opposite behaviour, with the higher density at the velocity peak and the lower densities in the line wings. The analysis performed in these knots located far from the source could, however, be affected by a not-perfect alignment of the slit with the jet axis and by the intrinsic jet width larger than the slit, as discussed in Sect. 3.1.1. Nevertheless, such a pattern agrees with the results obtained by Beck et al. (2007) using integral field spectroscopy of this part of the jet. They found that both the velocity and the electron density decrease with distance from the jet axis. Such behaviour is consistent with models for jet internal working surfaces.

\subsubsection{The $\mathrm{HH} 1$ jet}

In $\mathrm{HH} 1$, we define a LVC and a HVC from the [Fe II] $1.644 \mu \mathrm{m}$ line profile of knot KL. We selected a HVC from $\sim-80$ to $\sim-22 \mathrm{~km} \mathrm{~s}^{-1}$ and a LVC from $\sim-22$ to $\sim 36 \mathrm{~km} \mathrm{~s}^{-1}$. In analogy to HH34, we computed $n_{\mathrm{e}}$ for the LVC and the HVC separately for all extracted knots. Visual extinction and electron temperature values in each knot were taken from Nisini et al. (2005). At variance with the $\mathrm{HH} 34$ jet, the $n_{\mathrm{e}}$ in the LVC is lower than in the HVC. In the HVC, $n_{\mathrm{e}}$ decreases knot by knot from a value of $10^{4} \mathrm{~cm}^{-3}$ in knot KL, to $3.8 \times 10^{3} \mathrm{~cm}^{-3}$ in knot $\mathrm{F}$. On the other hand, in the LVC $n_{\mathrm{e}}$ decreases from $9.8 \times 10^{3} \mathrm{~cm}^{-3}$ to $8.3 \times$ $10^{3} \mathrm{~cm}^{-3}$ in knot KL and JI, respectively. These $n_{\mathrm{e}}$-values are in agreement with those derived by Nisini et al. (2005) in velocity integrated spectra.

The presence of two velocity components and the corresponding velocity dependence on electron density, was already found by Solf et al. (1991), who produced a PV diagram of the electron density in the HH1 jet using the optical [S II] $\lambda 6716 / 6731$ line ratio. They found a blue-shifted component with an electron density of $\sim 4000 \mathrm{~cm}^{-3}$ and a slightly redshifted component having a lower density of $\sim 1000-2000 \mathrm{~cm}^{-3}$. They interpreted the blue-shifted high-density component as due 


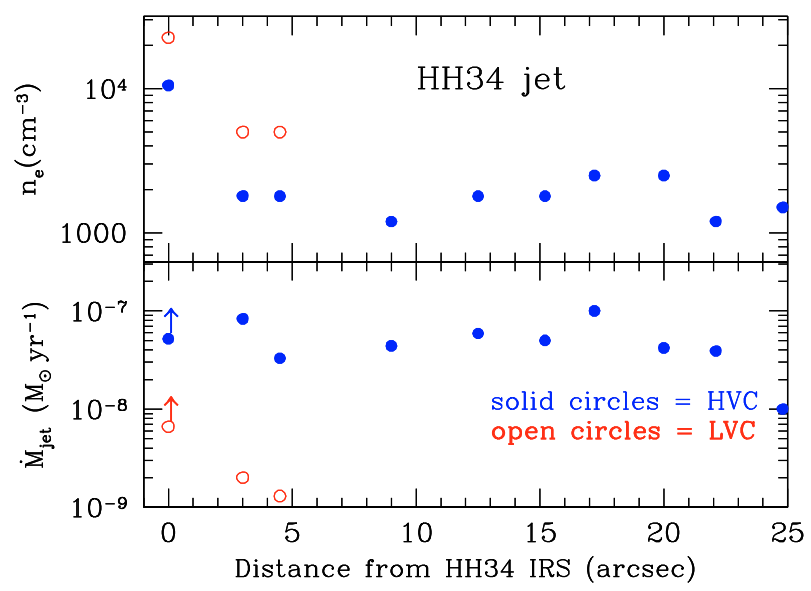

Fig. 11. The electron density (upper panel) and mass flux (bottom panel) are represented as a function of the distance from the source for the HH34 jet. Solid circles indicate the the electron density and mass flux for the HVC, while open circles refer to the values of the LVC.

to scattered light originating from a jet region closer to the star, where the density and excitation are higher. This interpretation is, however, not consistent with our IR observations. Indeed, the blue-shifted line component is clearly identifiable as the main jet component, extending at large distances from the exciting source. Moreover, our $\mathrm{H}_{2} \mathrm{PV}$ diagram (Fig. 6) clearly shows the presence of two separate components that cannot be attributed to scattered light contribution. The redshifted component peaks at the KL position and decreases in intensity further out, while the second blue-shifted component gradually increases its intensity with distance. One possible interpretation is that the redshifted component belongs to a different jet that intersects the HH1 jet at the KL position. Such a jet could be responsible for the two bright knots designated as HH501 objects by Reipurth et al. (2000), which are moving away from VLA1 with a proper motion vector inclined with respect to the $\mathrm{HH} 1$ jet by $\sim 10^{\circ}$. If we assume the axis of this separate jet equal to the direction of the proper motion vector, the HH501 jet should intersect the $\mathrm{HH} 1$ jet at the position of the KL knots.

\subsection{Mass flux}

The mass flux rate, $\dot{M}_{\text {jet }}$, along the beam of the HH34 and HH1 jets was recently measured in Podio et al. (2006) and Nisini et al. (2005), respectively, using different tracers, both optical ([S II], [O I $]$ ) and infrared ([Fe II $], \mathrm{H}_{2}$ ). These works show that the mass flux derived directly from the [Fe II] line luminosity, using the measured tangential velocity, is always equal or larger than the $\dot{M}_{\text {jet }}$ value derived from the luminosity of the optical atomic tracers in spite of the possibility that part of the iron is still locked on dust grains. This is probably due to the fact that [Fe II] traces a larger fraction of the total flowing mass than the optical lines, as discussed in Nisini et al. (2005). At the same time, it was found that in these jets the mass flux traced by the $\mathrm{H}_{2}$ molecular component is negligible with respect to the mass flux due to the atomic component. Taking advantage of the velocity resolved observations available, we now want to measure $\dot{M}_{\text {jet }}$ in the different velocity components and examine which of them is transporting more mass in the jet.

The $\dot{M}_{\text {jet }}$ value was obtained here from the luminosity of the [Fe II] $1.644 \mu \mathrm{m}$ line, adopting the relationship $\dot{M}=\mu m_{\mathrm{H}} \times$ $\left(n_{\mathrm{H}} V\right) \times v_{\mathrm{t}} / l_{\mathrm{t}}$, where $\mu=1.24$ is the average atomic weight; $m_{\mathrm{H}}$ and $n_{\mathrm{H}}$ are the proton mass and the total density; $V$ is the emitting region; and $v_{\mathrm{t}}$ and $l_{\mathrm{t}}$ are the velocity and length of the knot, projected perpendicular to the line of sight. The product $n_{\mathrm{H}} V$ can be expressed as $L$ (line) $\left(h v A_{\mathrm{i}} f_{\mathrm{i}} \frac{\mathrm{Fe}}{\mathrm{Fe}} \frac{[\mathrm{Fe}]}{\mathrm{H}}\right)^{-1}$ where $A_{\mathrm{i}}$ and $f_{\mathrm{i}}$ are the radiative rates and fractional population of the upper level of the considered transition and $\frac{\mathrm{Fe}^{+}}{\mathrm{Fe}}$ is the ionisation fraction of the iron having a total abundance with respect to hydrogen of $\left[\frac{\mathrm{Fe}}{\mathrm{H}}\right]$.

We have assumed that all iron is ionised, and has a solar abundance of $2.8 \times 10^{-5}$ (Asplund et al. 2005, i.e., no dust depletion). This latter hypothesis leads to a lower limit of the actual mass flux, since it has been shown that the velocity-averaged, gas-phase iron abundance in the $\mathrm{HH} 34$ and $\mathrm{HH} 1$ jets might be only between 20 and $70 \%$ of the solar value (Nisini et al. 2005; Podio et al. 2006). We do not adopt the Fe abundance estimate provided in these papers, since we do not know if the depletion pattern is constant among the LVC and HVC.

To compute the fractional population, we have used the $n_{\mathrm{e}}$ values derived separately for the LV and HV components, while we have assumed a single temperature for both components, equal to the values derived in Nisini et al. (2005) for the $\mathrm{HH} 1$ jet and a constant value of $7000 \mathrm{~K}$ for HH34 given by the average value derived by Podio et al. (2006) in knots from E to I.

Tangential velocity values have been derived from the radial velocities assuming an inclination angle $i=10^{\circ}$ for $\mathrm{HH} 1$ and $i=$ $22^{\circ} 7 \pm 5^{\circ}$ for HH34 (Bally et al. 2002; Eislöffel \& Mundt 1992). In the case of $\mathrm{HH} 34$, the inclination angle derived by Eislöffel \& Mundt (1992) is preferred to the value of $i=30^{\circ}$ estimated by Heathcote \& Reipurth (1992), who did not consider the pattern motion of the jet knots. The resulting tangential velocities of the HVC derived here are listed in Table 4. Compared to the values derived by the proper motion analysis of the [S II] emission by Reipurth et al. (2002), we derive smaller velocities in the internal knots (by $\sim 20-50 \mathrm{~km} \mathrm{~s}^{-1}$ in knots A6-A3) and larger velocities in the more distant knots. In particular, we see no evidence for a deceleration of the jet as shown by Reipurth et al. (2002), and the velocity remains higher than $200 \mathrm{~km} \mathrm{~s}^{-1}$ along the jet length. This could be an indication either that the inclination angle does not remain constant along the jet, or that the [Fe II] line has a kinematical behaviour different from [S II].

The luminosity of the line has been computed by integrating the extinction corrected flux of the knot in the same range of velocity used to calculate the electronic density. In Tables 4 and 5, the derived values, together with the parameters adopted, are reported for the HH34 and HH1 jets. In Fig. 11, the HH34 $\dot{M}_{\text {jet }}$ is plotted for the HVC and LVC as a function of the distance from the central source.

In both jets, $\dot{M}_{\text {jet }}\left(\right.$ LVC) is lower than $\dot{M}_{\text {jet }}(\mathrm{HVC})$ by a factor of about 6 (in HH1) and 10 (in HH34). In HH34, the $\dot{M}_{\text {jet }}(\mathrm{HVC}) / \dot{M}_{\text {jet }}(\mathrm{LVC})$ ratio does not reflect the derived $n_{\mathrm{e}}$ ratio in the two components, indicating that the $\left(n_{\mathrm{H}} V\right) \times v_{\mathrm{t}}$ product is significantly smaller in the LVC (around 8 times) than in the HVC. Since the difference in tangential velocity between each component is not enough to justify such a result, the larger $\dot{M}_{\text {jet }}(\mathrm{HVC})$ indicates a significant higher $\left(n_{\mathrm{H}} V\right)$ product in the HVC, i.e., the HVC has either an emission volume or a total density larger than the LVC, or both. This result could be biased by the finite slit width with respect to the jet diameter. In fact, the jet width measured only by HST in the optical lines is less than 0.3 up to a distance of $5^{\prime \prime}$ from the source (Reipurth et al. 2002). In addition, the jet emission in the LVC could be broader than the jet width derived from velocity-integrated emission maps, as observed, e.g., in DG Tau (Bacciotti et al. 2000). This effect propably does not account for the difference of nearly two orders 
of magnitude in derived mass-loss rates for the LVC and $\mathrm{HVC}$ in the inner regions of $\mathrm{HH} 34$, but the derived mass-loss rates for the LVC are likely lower limits. We also note that the reported values of $\dot{M}_{\text {jet }}(\mathrm{HVC})$ remain roughly constant $\left(\sim 5 \times 10^{-8} M_{\odot} / \mathrm{yr}\right)$ along the whole jet as expected in steady jet flows. This fact excludes significant flux losses as the jet opening angle increases. In jets from CTTSs, Hartigan et al. (1995) have already shown that the mass flux in the LVC should be lower than in the HVC: from the analysis of not spatially-resolved optical spectra, they concluded that the LVC in jets from CTT stars could be responsible for carrying the majority of mass and momentum only if the emitting region were smaller than $1 \mathrm{AU}$.

In HH34, the mass flux derived for the internal knot A6 is very low, much smaller than the $\dot{M}_{\text {jet }}$ value in knot A3. This is likely due to the fact that we assumed here the same extinction value of $7.1 \mathrm{mag}$ as estimated by Podio et al. (2006) as an average over the entire knot A, i.e., over $4^{\prime \prime}$. The extinction value on-source has been estimated to be about 45 mag (Antoniucci et al. 2008): therefore, a large dust column density gradient is expected in the inner jet region. Assuming an $A_{\mathrm{V}}$ lower limit of $7.1 \mathrm{mag}$ also in the red-shifted component rA, we derive here a lower limit for $\dot{M}_{\text {jet }}$ of $\sim 1 \times 10^{-9} M_{\odot} \mathrm{yr}^{-1}$. The same problem probably applies to the internal knot KL in HH1, where the 8.3 mag of extinction are the average value in the whole jet region LI.

We finally note that while the $\dot{M}_{\text {jet }}$ determined here for the HH34 jet agrees fairly well with the velocity integrated values estimated in Podio et al. (2006), there is some disagreement between the $\dot{M}_{\text {jet }}$ derived for HH1 here and in Nisini et al. (2005). The larger discrepancy is being found in the internal JI knots, where Nisini et al. (2005) report a $\dot{M}_{\text {jet }}$ a factor of six larger. Part of the disagreement is due to the different adopted tangential velocity. The largest discrepancy is, however, due to a smaller flux (a factor of three) measured in the ISAAC $0 .{ }^{\prime} 3$ slit with respect to the $1^{\prime \prime}$ slit used in Nisini et al. (2005). This latter measurement might have been contaminated by the presence of the second jet responsible for the redshifted velocity component or the $\mathrm{HH} 1$ jet itself might have a diameter wider than 0.3 .

It is interesting to compare the derived mass flux rates with the source mass accretion rate, to estimate the $\dot{M}_{\text {jet }} / \dot{M}_{\text {acc }}$ efficiency in embedded young sources. Antoniucci et al. (2008) have derived a mass accretion rate of the order of $4.1 \times$ $10^{-6} M_{\odot} \mathrm{yr}^{-1}$ for HH34 IRS. This would imply $\dot{M}_{\text {jet }} / \dot{M}_{\text {acc }} \geq 0.01$, in agreement with what is found in T Tauri stars and predicted by MHD jet launching models (e.g., Ferreira et al. 2006). There are no measurements of the mass accretion rate in VLA1, the exciting source of the HH1 jet, available. We can give a rough estimate of this value by assuming that the source bolometric luminosity $\left(L_{\mathrm{bol}} \sim 50 L_{\odot}\right.$, Chini et al. 1997) is entirely due to accretion. Such an assumption is supported by the fact that VLA1 is a known class 0 source. If we further assume a stellar mass and radius of $1 M_{\odot}$ and $3 R_{\odot}$ respectively, we get $\dot{M}_{\text {acc }} \sim 6 \times$ $10^{-6} M_{\odot} \mathrm{yr}^{-1}$, which would imply in this case $\dot{M}_{\text {jet }} / \dot{M}_{\text {acc }} \geq 0.007$.

\section{3. $\mathrm{The}^{+} / \mathrm{Fe}^{+}$ratio in $\mathrm{HH} 1$}

We have detected forbidden [Ti II] emission lines for the first time in HH1. Similar to iron, Ti has a low-ionisation potential, of only $6.82 \mathrm{eV}$, and thus is expected to be fully ionised in the jet plasma. The two detected lines have excitation energies of the order of $7000 \mathrm{~K}$ and critical densities, at $10000 \mathrm{~K}$, of $\sim 5 \times$ $10^{4} \mathrm{~cm}^{-3}$. They have similar excitations, thus, with respect to [Fe II] IR lines. Titanium, as iron, is a highly-refractory element, thus the ratio of $[\mathrm{Ti} \mathrm{II}] /[\mathrm{Fe} \mathrm{II}]$ lines can give some clue as to the relative gas-phase abundances of $\mathrm{Ti}$ and $\mathrm{Fe}$, and, in turn, as to the selective depletion of these elements on dust grains still present in the jet beam. Nisini et al. (2005) have indeed shown that the gas-phase abundance of Fe in HH1 is lower than the solar value, in particular in the inner jet regions where it is only between $20-30 \%$ of the solar Fe abundance. This indicates that part of the iron is depleted on grains and, consequently, that a significant fraction of dust is present in the jet beam. We can check here if titanium follows the same depletion pattern by comparing the observed [Ti II] $2.160 \mu \mathrm{m} /[\mathrm{Fe}$ II] $1.644 \mu \mathrm{m}$ ratio with the value theoretically expected assuming solar abundance values given by Asplund et al. (2005), i.e., $[\mathrm{Fe} / \mathrm{Ti}]_{\odot}=354$. For this analysis, we have performed a statistical equilibrium calculation taking the [Ti II] radiative transition rates and electron collisional rates calculated in Bautista et al. (2006, Bautista, private communication), and considering the temperature and density values measured in each HH1 knot in Nisini et al. (2005). The [Ti II] $2.160 \mu \mathrm{m} /[\mathrm{Fe}$ II] $1.644 \mu \mathrm{m}$ intensity ratio is rather insensitive to the adopted physical conditions, being of the order of $\sim 500$ in a temperature range from 9000 to $12000 \mathrm{~K}$, and density range from $5 \times 10^{3}$ to $1.2 \times 10^{4} \mathrm{~cm}^{-3}$. The observed ratios range instead between 150 (knot JI) and 280 (knot G), implying a gas-phase $\mathrm{Fe} / \mathrm{Ti}$ ratio $2-3$ times lower than solar. Thus, there is an overabundance of $\mathrm{Ti}$ in the gas phase, with respect to $\mathrm{Fe}$, relative to the solar value. This result indicates that the release of gas-phase elements from dust grains likely follows a selective pattern in which Ti-bearing condensates are more easily destroyed. A large $\mathrm{Ti} / \mathrm{Fe}$ abundance ratio relative to solar abundances has been observed previously in the ejecta of $\eta$ Carinae by Bautista et al. (2006) who suggest two different scenarios to explain this finding: either there is a spatial separation between Ti- and Fe-bearing condensates in the same dust grain, which makes the titanium more exposed to evaporation; or Ti-bearing grains are smaller than the Fe-bearing grains and thus more easily destroyed. Studies of this kind, employing a larger number of refractory species, seem therefore promising to constrain the composition and structure of dust grains in different environments.

\section{HH34 small scale jet: comparison with models}

The [Fe II] PV observed in HH34 is similar to the ones observed in some CTTSs, such as HL Tau and RW Aurigae (Pyo et al. 2006). In these latter, as in other CTTSs observed in the optical, the HVC peak has an offset with respect to the central source, of typically 50-100 AU. Given the larger distance of HH34 with respect to the sources in Taurus, we are not able to resolve such spatial regions, and the HV peak in our PV is centred on the source. In CTT jets, the LVC is usually confined within $d \lesssim$ $200 \mathrm{AU}$ from the source. In HH34, on the contrary, the LVC at $-60 \mathrm{~km} \mathrm{~s}^{-1}$ persists at larger distances, up to 1000-2000 AU. In the following, we discuss these findings in light of current theoretical models for the production of YSO jets.

Magneto-centrifugal jet launching models, such as the discwind and X-wind models (Ferreira 1997; Shang et al. 1998; Garcia et al. 2001; Pudritz et al. 2007) predict the presence of a broad velocity range close to the source position, corresponding to the uncollimated outer streamlines. In both these models, however, the LVC is confined to a relatively small region at $d \lesssim 200 \mathrm{AU}$ from the source. Thus, the LV gas we observe farther away needs to be locally heated, probably by shocks. Within $400 \mathrm{AU}$ from the HH34 IRS source the line emission decreases with velocity almost continuously from its peak at $\sim-100 \mathrm{~km} \mathrm{~s}^{-1}$ to redshifted velocities up to $\sim+200 \mathrm{~km} \mathrm{~s}^{-1}$. 
We can compare the kinematical signatures we observe in this region with those in the synthetic PV diagrams available for the different jet models. Synthetic PV diagrams from X-wind models have been constructed for optical [S II] and [O I] lines (Shang et al. 1998), while [Fe II] PV plots have been presented by Pesenti et al. (2003) for the cold disc-wind model of Ferreira (1997).

The cold disc-wind synthetic P-V diagram presented in Pesenti et al. (2003) was constructed assuming a spatial sampling and spectral resolution similar to those of our observations. This diagram predicts [Fe II] $1.64 \mu \mathrm{m}$ maximum (de-projected) velocities of the order of $-700 \mathrm{~km} \mathrm{~s}^{-1}$. In contrast, the maximum velocity measured in HH34 from the FWZI in knot A6 is $\sim-400 \mathrm{~km} \mathrm{~s}^{-1}$. However, the observed range of line of sight velocities predicted by disc-wind models significantly depends on the assumed range of launching radii and magnetic lever arm parameter $\left(\lambda=\left(r_{\mathrm{A}} / r_{0}\right)^{2}\right.$, with $r_{\mathrm{A}}$ and $r_{0}$, the Alfvén and launch radii, respectively). Ferreira et al. (2006) have shown that cold disc-wind models in general predict overly large line of sight velocities with respect to the observations because they have overly large magnetic lever arms of the order of 50 or higher. On the other hand, warm disc wind solutions with magnetic lever arms in the range 2-25 predict lower poloidal velocities, in better agreement with observations (Ferreira et al. 2006). In particular, our measured poloidal velocities of the order of $250 \mathrm{~km} \mathrm{~s}^{-1}$, estimated from the observed peak radial velocity corrected for the inclination angle, would imply a value of $\lambda$ in the range 4-8, for launching radii in the range 0.07-0.15 AU (Ferreira et al. 2006).

$\mathrm{X}$-wind models predict a narrower spread in velocity, which agrees with our observed range, taking into account the inclination angle of $23^{\circ}$ of the HH34 flow. In such models, however, the intermediate and low-velocity components are predicted to be present only in the inner $\sim 30$ AU from the central source, in contrast with observations. In addition, the synthetic PV diagrams constructed by Shang et al. (1998) do not include heating processes in the calculation, but assume a uniform ionisation fraction and electron temperature.

Another interesting feature predicted by $\mathrm{X}$-wind models is the presence of a significant redshifted emission at the stellar position similar to what we observe in HH34. This is the first case where such a feature is observed, and indeed the absence of the redshifted emission in the PV of other CTT jets was taken as an indication to rule out the $\mathrm{X}$-wind model for these sources (Takami et al. 2006). In HH34, such redshifted emission was not detected in previous observations probably because of the lack of sensitivity.

In conclusion, a qualitative comparison based on the available synthetic PV diagrams indicates that both $\mathrm{X}$-wind and discwind models reproduce the kinematics observed in the HH34 jet at its base, while none of them can reproduce the large scale LVC observed at very large distances from the source. A more quantitative comparison would require the construction of [Fe II] synthetic $\mathrm{P}-\mathrm{V}$ diagrams exploring a larger range of parameters and heating mechanisms.

The other observational finding in our study that can be compared to model predictions is the dependence of the electron density and mass flux on velocity. The fact that the electron density is higher in the LVC than in the HVC is neither consistent with the available $\mathrm{X}$-wind nor with disc-wind models. In discwind models, the high velocity and dense gas ejected in the inner streamlines is bracketed by the slower gas at lower density originating from more external and less excited regions of the disc. Cabrit et al. (1999) show, in fact, that the electron density increases toward the axis of the flow. Similarly, in X-wind models the electron density and ionisation decreases with the distance from the central axis (Shu et al. 1995). We point out that the main parameter predicted by different jet launching models is not the electron density, but the total density and its variation with velocity. Therefore, the found inconsistency may stem from an incorrect modelling of the excitation mechanism responsible for the gas ionisation, not necessarily from a wrong underlying MHD solution.

An alternative possibility is that the LVC represents gas not directly ejected in the jet, but dense ambient gas entrained by the high-velocity collimated jet. Observations at higher spatial resolution would be needed to perform an analysis similar to that presented here for the gas within 200 AU from the source. This would allow us to isolate as much as possible the component of gas ejected at low velocity in the jet itself.

\section{1. $\mathrm{H}_{2} H V C$ and $L V C$ in $H H 34$}

High- and low-velocity $\mathrm{H}_{2}$ emission within a few hundred $\mathrm{AU}$ from the driving source has been detected in a dozen outflows (Davis et al. 2001); such emission regions have been called molecular hydrogen emission-line region (MHEL) in analogy to the atomic FELs regions observed in T Tauri stars. With respect to FEL regions, the gas components associated with MHELs usually show lower radial velocities and velocity spreads.

In light of these previous findings, there are two scenarios that can explain the characteristics of the $\mathrm{LVC}$ in $\mathrm{H}_{2}$ lines in HH34. The first hypothesis is that such a low-velocity warm molecular gas is excited by oblique shocks occurring at the wall of a cavity created by the interaction of a wide angle wind with the ambient medium. Indeed, the large reflection nebulosity associated with HH34 IRS suggests the presence of a cavity illuminated by the central source. The second possibility is that the $\mathrm{H}_{2}$ gas originates from the external layers of a discwind. Magneto-centrifugal wind models, in fact, predict that the fast collimated inner component coexists with a slow and wide external component at low excitation. In a previous study, the $\mathrm{H}_{2}$ emission observed within 100 AU from DG Tau has been interpreted by Takami et al. (2004) in this scenario. Modelling of the $\mathrm{H}_{2}$ molecule survival and excitation in disc-winds is, however, needed to support this interpretation.

The fact that the $\mathrm{H}_{2} \mathrm{HVC}$ is observed only from a distance of $\sim 2^{\prime \prime}$ from the star, at variance with [Fe II], may indicate that the molecular gas does not survive in conditions of highly-excited gas constituting the high-velocity inner jet region. In this inner region, the jet is probably travelling inside a low-density cavity and thus no molecular material is present to be entrained by the fast moving flow. Reformation of $\mathrm{H}_{2}$ in the jet could be at the origin of the $\mathrm{H}_{2} \mathrm{HVC}$ seen further out: we point out that dust in the jet is not fully destroyed (Podio et al. 2006) and thus the $\mathrm{H}_{2}$ molecules can be formed again once the physical conditions become favourable for the molecule survival. Excitation in the wings of mini-bow shocks along the flow can be another possible explanation for the $\mathrm{H}_{2} \mathrm{HVC}$, although the high radial velocity of $\mathrm{H}_{2}$ close to the velocity of the atomic gas, is not supported by $\mathrm{H}_{2}$ excitation models in bow-shocks (Flower et al. 2003).

\section{Conclusions}

We have presented $H$ and $K$-band spectra of the HH34 and $\mathrm{HH} 1$ jets, where [Fe II], $\mathrm{H}_{2}$ and for the first time [Ti II] emission lines have been detected. These observations provide us 
with detailed information about the kinematics of the emitting gas allowing us to resolve two velocity components in each jet and to measure their physical parameters separately. From the [Fe II] $1.600 \mu \mathrm{m} / 1.644 \mu \mathrm{m}$ ratio the electron density along both jets and for each velocity component has been derived. In addition, the mass flux has been inferred from the [Fe II] $1.644 \mu \mathrm{m}$ line at different positions along the jet axis and for both velocity components. Finally, the [Ti II $] /[\mathrm{Fe}$ II] ratio gives us important information about the mechanism of dust reprocessing. We summarise our results as follows:

- In HH34, the atomic and molecular gas show two velocity components, the so-called HVC and LVC near the source. We also detect for the first time the fainter red-shifted counterflow down to the central source and up to a distance of $25^{\prime \prime}$ from the star.

- HH1 is traced down to $\sim 1^{\prime \prime}$ from the source. The kinematics of the region closest to the driving source is again characterised by a double velocity component, one blue-shifted and one red-shifted with respect to the source LSR. We interpret the red-shifted component as part of another jet, previously detected by Reipurth et al. (2000) in optical and near-IR lines.

- In the innermost HH34 jet region, the electron density increases as the velocity of the jet decreases, with average values of $10.8 \times 10^{3} \mathrm{~cm}^{-3}$ and $4.7 \times 10^{3} \mathrm{~cm}^{-3}$ for the LV and HV components, respectively. On the contrary, at large distance from the source, in HH34 as well as in HH1, $n_{\mathrm{e}}$ increases with velocity. The average values of the electron density along $\mathrm{HH} 1$ are $n_{\mathrm{e}} \sim 9.8 \times 10^{3} \mathrm{~cm}^{-3}$ and $n_{\mathrm{e}} \sim 9 \times 10^{3} \mathrm{~cm}^{-3}$ for the blue- and red-shifted components, respectively.

- The mass flux is mainly carried by the high-velocity gas in both jets. We derive a lower limit on the mass flux of $3-8 \times$ $10^{-8} M_{\odot} \mathrm{yr}^{-1}$ along the HH34 and HH1 jets.

- Comparing the observed [Ti II]/[Fe II] ratio with the theoretical one, we derived a gas-phase $\mathrm{Fe} / \mathrm{Ti}$ abundance ratio 2-3 times less than solar. This seems to indicate that the release of gas-phase elements from dust grains likely follows a selective pattern in which Ti-bearing condensates are more easily destroyed than the Fe ones.

- By a comparison of our PV diagrams and electron densities with those provided by models for MHD jet launching, we suggest that the kinematical features observed close to the source in our spectra can be, qualitatively, reproduced by both disc-wind and X-wind models, although neither of them is able to explain the persistency of the LVC at large distances (up to $1000 \mathrm{AU}$ ) from the launching region. Moreover, none of the excitation mechanisms proposed in these models can explain the velocity dependence on electron density that we measure in HH34. Alternatively, the LVC of the [Fe II] lines that we observe in HH34 could represent dense gas entrained by the high-velocity collimated jet.

- In the $\mathrm{H}_{2}$ line, only the LVC is observed down to the central source, while the HVC is detected only up to $2^{\prime \prime}$ from the star. We suggest that the low-velocity molecular gas could be excited by oblique shocks occurring along the wall of a cavity created by the interaction of a wide angle wind or by the external layers of a disc-wind. On the other hand, the HVC of the $\mathrm{H}_{2}$ emission could be due to reformation of $\mathrm{H}_{2}$ molecules along the jet, since the dust in the jet is probably not fully destroyed.

Acknowledgements. The present work was partly supported by the European Community's Marie Curie Actions - Human Resource and Mobility within the JETSET (Jet Simulations, Experiments and Theory) network under contract MRTN-CT-2004 005592. We thank Manuel Bautista for having provided us with the atomic data for the [Ti II] statistical equilibrium model.

\section{References}

Anglada, G., Estalella, R., Mauersberger, R., et al. 1995, ApJ, 443, 682 Antoniucci, S., Nisini, B., Giannini, T., \& Lorenzetti, D. 2008, A\&A, 479, 503 Asplund, M., Grevesse, N., \& Sauval, A. J. 2005, in Cosmic Abundances as Records of Stellar Evolution and Nucleosynthesis, ed. T. G. Barnes, III, \& F. N. Bash, ASP Conf. Ser., 336, 25

Bacciotti, F., Mundt, R., Ray, T. P., et al. 2000, ApJ, 537, L49

Bally, J., Heathcote, S., Reipurth, B., et al. 2002, AJ, 123, 2627

Bautista, M. A., Hartman, H., Gull, T. R., Smith, N., \& Lodders, K. 2006, MNRAS, 370, 1991

Beck, T. L., Riera, A., Raga, A. C., \& Reipurth, B. 2007, AJ, 133, 1221

Cabrit, S., Ferreira, J., \& Raga, A. C. 1999, A\&A, 343, L61

Camenzind, M. 1990, in Rev. Mod. Astron., ed. G. Klare, 3, 234

Chini, R., Reipurth, B., Sievers, A., et al. 1997, A\&A, 325, 542

Chini, R., Ward-Thompson, D., Kirk, J. M., et al. 2001, A\&A, 369, 155

Choi, M., \& Zhou, S. 1997, ApJ, 477, 754

Davis, C. J., Smith, M. D., \& Eislöffel, J. 2000, MNRAS, 318, 747

Davis, C. J., Ray, T. P., Desroches, L., \& Aspin, C. 2001, MNRAS, 326, 524

Davis, C. J., Whelan, E., Ray, T. P., \& Chrysostomou, A. 2003, A\&A, 397, 693

Davis, C. J., Nisini, B., Takami, M., et al. 2006, ApJ, 639, 969

Eislöffel, J., \& Mundt, R. 1992, A\&A, 263, 292

Eislöffel, J., Mundt, R., \& Böhm, K.-H. 1994, AJ, 108, 1042

Eislöffel, J., Smith, M. D., \& Davis, C. J. 2000, A\&A, 359, 1147

Ferreira, J. 1997, A\&A, 319, 340

Ferreira, J., Dougados, C., \& Cabrit, S. 2006, A\&A, 453, 785

Flower, D. R., Le Bourlot, J., Pineau des Forêts, G., \& Cabrit, S. 2003, MNRAS, 341, 70

Garcia, P. J. V., Ferreira, J., Cabrit, S., \& Binette, L. 2001, A\&A, 377, 589

Hamann, F., Simon, M., Carr, J. S., \& Prato, L. 1994, ApJ, 436, 292

Hartigan, P., Raymond, J., \& Hartmann, L. 1987, ApJ, 316, 323

Hartigan, P., Morse, J. A., \& Raymond, J. 1994, ApJ, 436, 125

Hartigan, P., Edwards, S., \& Ghandour, L. 1995, ApJ, 452, 736

Heathcote, S. \& Reipurth, B. 1992, AJ, 104, 2193

Hirth, G. A., Mundt, R., \& Solf, J. 1997, A\&AS, 126, 437

Lavalley-Fouquet, C., Cabrit, S., \& Dougados, C. 2000, A\&A, 356, L41

Matt, S., \& Pudritz, R. E. 2005, ApJ, 632, L135

Nisini, B., Caratti o Garatti, A., Giannini, T., \& Lorenzetti, D. 2002, A\&A, 393, 1035

Nisini, B., Bacciotti, F., Giannini, T., et al. 2005, A\&A, 441, 159 (N05)

Pesenti, N., Dougados, C., Cabrit, S., et al. 2003, A\&A, 410, 155

Podio, L., Bacciotti, F., Nisini, B., et al. 2006, A\&A, 456, 189 (P06)

Pudritz, R. E., Ouyed, R., Fendt, C., \& Brandenburg, A. 2007, in Protostars and Planets V, ed. B. Reipurth, D. Jewitt, \& K. Keil, 277

Pyo, T.-S., Hayashi, M., Kobayashi, N., et al. 2002, ApJ, 570, 724

Pyo, T.-S., Kobayashi, N., Hayashi, M., et al. 2003, ApJ, 590, 340

Pyo, T.-S., Hayashi, M., Kobayashi, N., et al. 2006, ApJ, 649, 836

Raga, A., \& Noriega-Crespo, A. 1998, AJ, 116, 2943

Raga, A. C., Velázquez, P. F., Cantó, J., \& Masciadri, E. 2002, A\&A, 395, 647

Reipurth, B., Heathcote, S., Yu, K. C., Bally, J., \& Rodríguez, L. F. 2000, ApJ, 534,317

Reipurth, B., Heathcote, S., Morse, J., Hartigan, P., \& Bally, J. 2002, AJ, 123, 362

Shang, H., Shu, F. H., \& Glassgold, A. E. 1998, ApJ, 493, L91

Shang, H., Glassgold, A. E., Shu, F. H., \& Lizano, S. 2002, ApJ, 564, 853

Shu, F. H., Najita, J., Ostriker, E. C., \& Shang, H. 1995, ApJ, 455, L155

Solf, J., Raga, A. C., Boehm, K. H., \& Noriega-Crespo, A. 1991, AJ, 102, 1147

Takami, M., Chrysostomou, A., Ray, T. P., et al. 2004, A\&A, 416, 213

Takami, M., Chrysostomou, A., Ray, T. P., et al. 2006, ApJ, 641, 357 